\title{
On the influence of forced homogeneous-isotropic turbulence on the settling and clustering of finite-size particles
}

\author{
Agathe Chouippe* and Markus Uhlmann ${ }^{\dagger}$ \\ Institute for Hydromechanics, Karlsruhe Institute of Technology (KIT), 76131 Karlsruhe, Germany \\ September 12, 2018, Manuscript accepted for publication in Acta Mechanica
}

\begin{abstract}
We investigate the motion of heavy particles with a diameter of several multiples of the Kolmogorov length scale in the presence of forced turbulence and gravity, resorting to interfaceresolved direct numerical simulation based on an immersed boundary method. The values of the particles' relative density (1.5) and of the Galileo number (180) are such that strong wakeinduced particle clustering would occur in the absence of turbulence [56]. The forced turbulence in the two present cases (with Taylor-scale Reynolds number 95 and 140) would lead to mild levels of clustering in the absence of gravity [55]. Here we detect a tendency to cluster with an intensity (quantified via the standard deviation of the distribution of Voronoï cell volumes) which is intermediate between these two limiting cases, meaning that forced background turbulence decreases the level of clustering otherwise observed under ambient settling. However, the clustering strength does not monotonously decay with the relative turbulence intensity. Various mechanisms by which coherent structures can affect particle motion are discussed. It is argued that the reduced interaction time due to particle settling through the surrounding eddy (crossing trajectories) has the effect of shifting upwards the range of eddies with a time scale matching the characteristic time scale of the particle. In the present cases this shift might bring the particles into resonance with the energetic eddies of the turbulent spectrum. Concerning the average particle settling velocity we find very small deviations (of the order of one percent) from the value obtained for an isolated particle in ambient fluid when defining the relative velocity as an apparent slip velocity (i.e. as the difference between the averages computed separately for the velocities of each phase). This is consistent with simple estimates of the non-linear drag effect. However, the relative velocity based upon the fluid velocity seen by each particle (computed via local averaging over a particle-attached sphere) has on average a smaller magnitude (by 5-7\%) than the ambient single-particle value.
\end{abstract}

\section{Introduction}

Many fluidic systems in geophysics and engineering involve suspended mobile particles of some kind. Prominent examples are droplets in atmospheric clouds and plankton in ocean currents, while a large number of technical applications can be found practically across all engineering disciplines.

Among the many relevant aspects of fluid-mediated particle motion, the settling velocity of heavy particles in a turbulent flow is of particular interest. While this quantity is clearly impor-

\footnotetext{
*agathe.chouippe@kit.edu

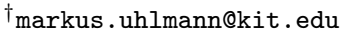


tant in various fields of research, it has received much attention in the meteorological community due to its relevance in precipitation forecasting [24, 48]. The available data, which will be reviewed in more detail below, shows that as a result of the interaction with a turbulent flow field the mean particle settling velocity can be significantly enhanced or reduced when compared to the reference value of a single particle settling in ambient fluid.

In many cases (e.g. small water droplets in atmospheric clouds) the particle size is much smaller than the smallest (i.e. Kolmogorov) flow scales and the flow at the particle scale can be considered as Stokesian. This scenario has been relatively well explored in the past, in particular through Eulerian-Lagrangian point-particle simulations. Conversely, when the particle diameter exceeds the Kolmogorov length scale and the Reynolds number of the flow around the particles is not negligible, it becomes necessary to consider the fluid-solid interaction problem at the level of each "finite-size" particle in all details. This more complex situation is the subject of the present work. Systems where finite-size effects become important arise for instance in meteorology when focusing on larger hydrometeors (e.g. hailstones), or in many chemical engineering processes. Across the large parameter space of the fluid-particle system a rich set of features emerges due to the action of turbulence, buoyancy, the collective action of particles, and the interplay between these different actors.

Let us first consider fluid-particle systems in the absence of gravity or buoyancy. For this configuration it is now well established that the particle motion can be considerably affected by the action of turbulent coherent structures, leading to an inhomogeneous sampling of the flow field. In a multi-particle system, the disperse phase will then typically be non-randomly distributed in space, a phenomenon which is commonly referred to as "preferential concentration" [49].

A number of mechanisms has been proposed to account for the phenomenon of particle clustering in turbulence. According to the centrifugal mechanism dense particles will tend to be expelled from vortical flow regions and accumulate preferentially in strain-dominated regions. Through asymptotic expansion Maxey [37] has shown how this argument applies to the simplest point particle model in the limit of small Stokes number (i.e. the particle-to-fluid ratio of time scales). For this class of particles, the Stokes number is generally considered to be the significant parameter with respect to the tendency to form clusters [2, 26, 40]. However, Yoshimoto and Goto [62] have shown that the definition of the fluid time scale should probably take into account the entire range of available flow scales. Experimental support for this multi-scale view of particle clustering has recently been provided by the study of Sumbekova et al. [50] who have found no significant dependency of the cluster statistics upon the conventional Stokes number. Also based upon the analysis of Maxey [37] a scenario dubbed "sweep-stick" mechanism has been proposed by Goto and Vassilicos [23] and further elaborated by Coleman and Vassilicos [10]. Since the leading order velocity difference between point particles (in the small Stokes number limit) and the carrier flow is proportional to the fluid acceleration, particles can be expected to "stick" to zeroacceleration points, which is what has indeed been observed in DNS [10]. Concerning finite-size particles, the data from particle-resolved DNS of Uhlmann and Chouippe [55] suggests that the sweep-stick mechanism also applies to particles whose diameter exceeds the Kolmogorov scale, leading to small but significant levels of clustering in their work. Based upon a Lagrangian description of the relative motion between particle pairs Zaichik and Alipchenkov [64], Chun et al. [8] and Zaichik and Alipchenkov [65] have derived models for the particles' radial distribution function. This type of approach is non-local, since the history of the fluid velocity field seen by the particles enters the description [5, 25]. Note that the relevance of this non-local clustering mechanism for finite-size particles has not been verified to date.

Let us now turn to the case of an ensemble of particles settling in the absence of a priori turbulence. In this configuration a new mechanism for generating a non-trivial spatial particle distribution comes into play through the effect of wakes. Roughly speaking, at a sufficiently 
large particle Reynolds number the reduced drag acting upon a vertically trailing particle induces "drafting-kissing-tumbling" motion in particle pairs [19, 60], which - at least transiently - brings a particle pair closer together. Essentially the same wake-attraction effect is also active in manyparticle configurations, where it can lead to the formation of massive columnar clusters which reach a sustained statistically stationary state in particle-resolved direct numerical simulations [33, 34]. Uhlmann and Doychev [56] and Doychev [12] have shown that wake-induced clustering appears above a critical Galileo number, a parameter which can be understood as a particle Reynolds number with a velocity scale constructed from buoyancy. The critical value in these studies approximately coincides with the value at which a change in the wake structure (from axi-symmetric to double-threaded oblique) is observed for a single settling particle. As a consequence of wake-induced clustering the average particle settling velocity is significantly enhanced in the many-particle case as compared to a single isolated particle. The formation of clusters as well as an increase of the settling velocity have also been evidenced experimentally by Huisman et al. [28]. It should be noted that despite the absence of a priori turbulence, the particle-induced flow field exhibits quite considerable levels of turbulence intensity even in relatively dilute systems if the Galileo number is significant (e.g. the standard deviation of the vertical fluid velocity component exceeds one fourth of the particle settling velocity in the simulations of Uhlmann and Doychev [56, cf. their figure 6a]). However, the "pseudo-turbulence" induced by the settling particles is distinct from classical turbulence, as it is characterized by a $(-3)$ power-law spectrum in terms of wavenumber [12, 36, 45].

Finally, let us return to the actual case of interest where heavy particles are settling in a homogeneous turbulent "background" flow. In this configuration we intuitively expect that for sufficiently high turbulence intensity the background flow will tend to suppress wake-induced cluster formation. On the other hand, the existence of gravity is expected to have a significant impact on the various mechanisms of preferential concentration discussed above. Wang and Maxey [58] have shown that turbulence leads to a significant enhancement of the average particle settling velocity in the framework of the point-particle model with Stokes drag and one-way coupling, a result which has subsequently been confirmed by various authors [4, 21, 31]. It has been observed by Wang and Maxey [58] and others that particles under these conditions preferentially sample the downward sweeping part of the vortical structures (an effect which is sometimes also referred to as "fast-tracking" [41]).

Aliseda et al. [1] have performed experiments with water droplets in decaying grid-turbulence in a wind-tunnel, where the particle diameter was much smaller than the Kolmogorov scale. They observed particle clustering and substantial enhancement of the average settling velocity, the latter increasing with the solid volume fraction. This is, therefore, clearly a collective effect, as opposed to the non-collective effect of Wang and Maxey [58] and others who have resorted to oneway coupled descriptions. Aliseda et al. [1] argue that the particle clusters (presumably formed through the centrifugal effect) settle at an increased speed due to the fact that the Reynolds number of the cluster is much larger than the particle Reynolds number. This interpretation has received support through the recent work of Monchaux and Dejoan [38] who have performed two-way coupled point-particle simulations with Stokes drag, resulting in a similar dependency of settling enhancement upon the solid volume fraction as in the experiments of Aliseda et al. [1].

Several experiments have observed a reduction in the average settling speed due to turbulence. Yang and Shy [61] have measured the motion of tungsten and glass particles with diameters in the range of $0.4 \ldots 4$ times the Kolmogorov scale in a water tank with oscillating grids, albeit at very low Reynolds number (the Taylor micro-scale Reynolds number was in the range of $20 \ldots 30$ ). They mostly observed an enhancement of the average settling velocity, except for their largest particles settling at the highest turbulence intensity for which a reduction by several percent was determined. Unfortunately these authors did not analyze particle clustering or pref- 
erential concentration. Good et al. [21] have performed measurements of sub-Kolmogorov size water droplets in homogeneous-isotropic air turbulence generated by a loudspeaker arrangement in a spherical enclosure. In this system the average settling velocity is reduced for the smallest relative turbulence intensity (i.e. for the largest and fastest settling particles).

It has long been recognized that a non-linear drag law can lead to a reduction of the average particle settling velocity in unsteady flows with zero mean $[41,52,58]$. In the framework of the one-way coupled point-particle approach, Good et al. [21] have shown that imposing a non-linear drag law (instead of the usual linear Stokes drag) can indeed lead to a reduction in settling speed, in accordance with what is measured in their companion experiments for the largest particles (which are still of sub-Kolmogorov size). Homann et al. [27] have performed particle-resolved DNS of a single sphere which steadily translates through homogeneous-isotropic turbulence. They observe a significant increase in the average drag coefficient, the increase scaling with the square of the relative turbulence intensity (i.e. normalized with the translation velocity). They also show that the observed scaling is consistent with the non-linear drag mechanism, at least for low to moderate values of this latter control parameter. Rosa et al. [46] have likewise investigated the effect of a non-linear drag law upon the settling behavior of one-way coupled point particles. Contrary to Good et al. [21], however, they did not find any reduction in the mean partice settling velocity, despite careful consideration of the numerical set-up (e.g. the turbulence forcing scheme). One possible cause for the observed discrepancy is the duration of the sampling interval, which was significantly longer in the study of Rosa et al. [46]. Chouippe and Uhlmann [7] have performed the first particle-resolved simulations of settling under forced homogeneous turbulence conditions. In their moderate-size systems a slight reduction of the average settling velocity has been observed for particles with a diameter equal to approximately 7 Kolmogorov lengths and Galileo number measuring 120 . While preferential concentration was not detected, it was found that the non-linear drag model of Homann et al. [27] indeed provides a reasonable prediction for the reduced settling speed.

Fornari et al. [17] and Fornari et al. [18] have performed particle-resolved DNS of forced homogeneous-isotropic turbulence with several hundred nearly neutrally-buoyant particles (solidto-fluid density ratio less than 1.04) with a diameter equivalent to approximately 12 Kolmogorov length scales. While particle clustering was not detected, they observed a reduction in the average settling velocity of up to $55 \%$ percent at the smallest value of the Galileo number (measuring 19), for which the relative turbulence intensity was approximately 5 times as large as the average settling velocity. Fornari et al. [17] perform a force analysis which shows that unsteady forces are significant in their parameter range. The analysis in the study of Fornari et al. [18] suggests that the horizontal velocity perturbations are mainly responsible for an average drag increase in this low-excess-density and high-turbulence-intensity case, hence causing the reduced settling speed.

Finally let us mention the only available experimental data (to our knowledge) on clustering of finite-size heavy particles in approximately homogeneous-isotropic turbulence. Fiabane et al. [16] have measured the positions of glass particles in water, while turbulence was forced through an array of impellers in an icosahedral enclosure. For particle diameters equivalent to 2 to 5 times the Kolmogorov length and a Galileo number of approximately 13 the authors detected a strong tendency to cluster. Unfortunately, however, the average settling velocity was not measured in that study.

In the present study we are considering heavy particles settling under gravity while a turbulent background flow is maintained via homogeneous-isotropic, large-scale, random forcing. We have performed interface-resolved DNS for two cases with much higher turbulent Reynolds number than previously investigated in Chouippe and Uhlmann [7], providing the largest-scale data-sets of this kind to date. The present study specifically builds upon the earlier work of Uhlmann and Doychev [56] for ambient settling and of Uhlmann and Chouippe [55] for particle- 
turbulence interaction without gravity by maintaining most parameters at similar values, while essentially varying the ratio between the turbulence intensity and the terminal particle velocity. In this fashion it is possible to analyze the particular influence of the relative turbulence intensity upon the particle settling behavior and upon their tendency to cluster.

The present manuscript is organized as follows. In section 2 we describe the numerical methodology and the set-up of the simulations. Section 3 is devoted to the presentation of the fluid phase statistics, while we analyze the settling velocity and the spatial distribution of the dispersed phase in detail in section 4 . The different mechanisms for cluster formation and modification of the particle settling velocity are discussed in section 5 before closing with a summary and outlook.

\section{Methodology}

We simulate the motion of multiple particles in forced homogeneous isotropic turbulence with the aid of the Immersed Boundary Method of Uhlmann [53] coupled to the turbulence forcing of Eswaran and Pope [13]. This configuration has been previously investigated at lower Reynolds number in Chouippe and Uhlmann [7] and in the absence of gravity in Uhlmann and Chouippe [55] while using the same methodology. The flow is governed by the incompressible NavierStokes equations (eq. 1 and 2) where $\mathbf{u}$ denotes the fluid velocity, $\rho_{f}$ and $v$ its density and kinematic viscosity (which are both supposed to be constant). The forcing term $\mathcal{F}$ in the momentum equation accounts for the turbulence forcing $\mathbf{f}^{(t)}$ and the influence of the particles on the flow $\mathbf{f}^{(i b m)}$.

$$
\begin{aligned}
\frac{\partial \mathbf{u}}{\partial t}+(\mathbf{u} \cdot \nabla) \mathbf{u}+\frac{1}{\rho_{f}} \nabla p & =v \nabla^{2} \mathbf{u}+\mathcal{F} \\
\nabla \cdot \mathbf{u} & =0 \\
\mathcal{F} & =\mathbf{f}^{(t)}+\mathbf{f}^{(i b m)}
\end{aligned}
$$

The solver employs a fractional step method with an implicit treatment of the viscous term (Crank-Nicolson) and a three step Runge Kutta method for the nonlinear terms. The spatial discretization relies on a second order finite differences method on a staggered mesh with a uniform discretization in the three directions. The forcing term $\mathbf{f}^{(\mathrm{ibm})}$ imposes the fluid velocity to be equal to the local particle velocity at the particle surface according to the Immersed Boundary Method of Uhlmann [53]. The motion of the particles is computed by the time integration of the Newton equation for the linear and angular motion. We focus here on very dilute regimes and treat collisions with a simple repulsive force mechanism [20]. The background flow is forced by the large scale turbulence forcing of Eswaran and Pope [13]. It consists in adding a forcing term $\mathbf{f}^{(t)}$ in the momentum equation that randomly forces the largest scales of the flow, and more specifically every wavenumber smaller than a given wavenumber $\kappa_{f}$. In the absence of buoyancy effects this would lead to the development of a homogeneous isotropic turbulent flow. For more details on the forcing method and its implementation in physical space in an IBM framework the reader is referred to [7].

The main physical parameters of the dispersed and continuous phases are given in the table 1 . The characteristics of the background flow are given in terms of the Reynolds number $R e_{\lambda}$, Kolmogorov length scale $\eta$ and velocity scale of the turbulence $u_{r m s}$. We use the following notation to distinguish between particle and fluid velocity. $\mathbf{u}=\left(u_{x}, u_{y}, u_{z}\right)^{T}$ refers to the fluid velocity and $\mathbf{v}^{(\mathbf{i})}=\left(v_{x}^{(i)}, v_{y}^{(i)}, v_{z}^{(i)}\right)^{T}$ to the velocity of ith the particle. We introduce the operator $\langle\cdot\rangle_{\Omega_{f}}$ corresponding to space-averaging over regions occupied by the fluid, and use the decomposition

$$
\mathbf{u}(\mathbf{x}, t)=\langle\mathbf{u}\rangle_{\Omega_{f}}(\mathbf{x}, t)+\mathbf{u}^{\prime}(\mathbf{x}, t)
$$




\begin{tabular}{rccccccccccc}
\hline case $G a$ & $D / \eta^{S P}$ & $D / \eta^{T P}$ & $R e_{\lambda}^{S P}$ & $R_{\lambda}^{T P}$ & $\Phi_{s}$ & $\rho_{p} / \rho_{f}$ & $u_{r m s}^{S P} / u_{g}$ & $u_{r m s}^{T P} / u_{g}$ & initial positions & source \\
\hline G178-R95 & 178 & 6.8 & 1.5 & 94.76 & 35.12 & 0.005 & 1.5 & 0.19 & 0.22 & final state of G178 present \\
G180-R140 & 180 & 8.5 & 2.6 & 142.2 & 64.37 & 0.005 & 1.5 & 0.29 & 0.31 & random & present \\
G178 & 178 & - & 0.69 & 0 & 170.2 & 0.005 & 1.5 & 0 & 0.23 & random & {$[56]$} \\
G0-R120 & 0 & 5.5 & 5.5 & 119.0 & 116.8 & 0.005 & 1.5 & $\infty$ & $\infty$ & random & {$[55]$} \\
R95 & - & - & - & 94.76 & - & 0 & - & - & - & - & present \\
R140 & - & - & - & 142.2 & - & 0 & - & - & - & - & {$[7]$} \\
R120 & - & - & - & 119 & - & 0 & - & - & - & - & {$[55]$} \\
\hline
\end{tabular}

Table 1: Main physical parameters of the cases investigated in the present study: Galileo number $G a$, length scale ratio $D / \eta$, Taylor-scale Reynolds number $R e_{\lambda}=\lambda u_{r m s} / \nu$, global solid volume fraction $\Phi_{s}$, density ratio $\rho_{p} / \rho_{f}$, and resulting velocity ratio $u_{r m s} / u_{g}$. For each quantity resulting from the flow characteristics $\left(\eta, R e_{\lambda}, u_{r m s}\right)$, the distinction is made between the value in single phase configuration and particle laden configuration with the respective SP and TP subscripts. Two types of initializations have been used for the particle positions: they are either randomly distributed in the domain or initialized with the final set of position of the case G178 which features columnar accumulation.

The dissipation $\varepsilon$ is then defined by

$$
\varepsilon(t)=2 v\left\langle S_{i j}^{\prime} S_{i j}^{\prime}\right\rangle_{\Omega_{f}}, \quad \text { with } S_{i j}=\frac{1}{2}\left(\frac{\partial u_{i}}{\partial x_{j}}+\frac{\partial u_{j}}{\partial x_{i}}\right),
$$

and the kinetic energy of the fluctuation $k$ as well as the characteristic velocity scale $u_{r m s}$ according to

$$
k(t)=\frac{1}{2}\left\langle\mathbf{u}^{\prime} \cdot \mathbf{u}^{\prime}\right\rangle_{\Omega_{f}}=\frac{3}{2} u_{r m s}^{2}
$$

From those quantities we define the Kolmogorov length scale $\eta=\left(v^{3} / \varepsilon\right)^{1 / 4}$, the Taylor microscale $\lambda=\left(15 v u_{r m s}^{2} / \varepsilon\right)^{1 / 2}$, the corresponding Reynolds number $R e_{\lambda}=\lambda u_{r m s} / \nu$, the largeeddy length scale $L=k^{3 / 2} / \varepsilon$, the large-eddy turnover time $\tau_{e}=u_{r m s}^{2} / \varepsilon$, the Kolmogorov time scale $\tau_{\eta}=(\nu / \varepsilon)^{1 / 2}$. We will make the distinction between scales related to the corresponding single phase simulation (which is obtained with exactly the same forcing conditions, but no particles) and scales of the particle laden configurations with the subscripts SP and TP respectively. In a single phase configuration the system would be parametrized by the Reynolds number $R e_{\lambda}$ while a multi-particle system requires additional four non-dimensional parameters. We propose to use the density ratio $\rho_{p} / \rho_{f}$ of the particle with respect to the fluid, the length scale ratio $D / \eta$ ( $D$ being the diameter of the particles) the solid volume fraction $\phi_{s}=N_{p} \mathcal{V}_{p} / \mathcal{L}_{x} \mathcal{L}_{y} \mathcal{L}_{z}$ (where $N_{p}$ is the number of particles, $\mathcal{V}_{p}=\pi D^{3} / 6$ their volume, and $\mathcal{L}_{i}$ the size of the domain in the direction $i$ ), and the Galileo number defined by $G a=u_{g} D / v$ with $u_{g}=\left(\left|\rho_{p} / \rho_{f}-1\right||\mathbf{g}| D\right)^{1 / 2}$ the gravitational velocity scale.

The Galileo number can be thought of as a Reynolds number built with $u_{g}$. In the case of one single spherical particle falling in a still fluid, its wake is known to follow different regimes according to the Galileo number: for the lowest Ga the particle fall straight and its wake is steady axisymmetric. Then when Ga increases the wake loses its axisymmetry to become planar symmetric and the particle follows an oblique path. This regime is known as the steady oblique regime. A further increase of the Galileo number will make the regime become unsteady with the oblique oscillating regime and followed then by the chaotic regime [32]. For a density ratio 


\begin{tabular}{rccc}
\hline Name & $\kappa_{f} / \kappa_{1}$ & $T_{L} v / \mathcal{L}_{x}^{2}$ & $\epsilon^{*} \mathcal{L}_{x}^{4} / v^{3}$ \\
\hline G178-R95 and R95 & 3.91 & $1.00 \times 10^{-4}$ & $5.14 \times 10^{8}$ \\
G180-R140 and R140 & 2.5 & $5.94 \times 10^{-5}$ & $3.52 \times 10^{9}$ \\
G0-R120 and R120 & 3.61 & $5.37 \times 10^{-5}$ & $1.31 \times 10^{9}$ \\
\hline
\end{tabular}

Table 2: Imposed parameters used for the forcing of the background turbulence with the method of [13] with the notation of [7]: forcing cutoff wavenumber $\kappa_{f}$ normalized by the smallest discrete wavenumber $\kappa_{1}$ in the horizontal direction, characteristic time of the random forcing $T_{L}$, dissipation-rate parameter $\varepsilon^{*}$

of $\rho_{p} / \rho_{f}=1.5$ and a Galileo number of 180 one particle would follow the steady oblique regime. In the case of multi-particle settling, for a solid volume fraction $\phi_{s}=0.005$ this Galileo number is characterized by the formation of columnar clusters and an enhancement of the settling velocity $[28,56]$.

In the current paper we consider three types of configurations: they can be either purely affected by the settling, or purely by the turbulence, or affected by the interaction of both settling and turbulence. The background turbulence is forced for the cases G178-R95, G180-R140 and G0R120 with a Galileo number of 178 and 180 for the cases G178-R95, G180-R140, and no influence of gravity $(G a=0)$ for G0-R120. In order to estimate the influence of the particle induced turbulence we also included the case G178 corresponding to multiple particle settling in an initially ambient flow. This case has been previously characterized in the former work of Uhlmann and Doychev [56], and is characterized by the formation of columnar clusters, large scale columnar fluid structures and an enhancement of the settling velocity. The parameters of the cases G178 and G178-R95 differ therefore only by the nature of the background turbulence: while cases G178 features only particle-induced turbulence (also called pseudo-turbulence [36, 44, 45]), the background flow of G178-R95 results in the interaction of the forced and the particle-induced turbulence. Cases G178-R95 and G180-R140 differ by the scales of the resulting forced turbulence that would be obtained in a single-phase configuration and consequently by the turbulence intensity $I$ which we define as the ratio between the characteristic velocity $u_{r m s}$ and settling velocity of one single particle falling in a still fluid.

The turbulence forcing is parametrized by the largest wavenumber forced, $\kappa_{f}$, by a timescale $T_{L}$ and a coefficient $\varepsilon^{*}$ which controls the amplitude of this forcing $[7,13]$. The coefficients used for the different cases are listed in a dimensionless form in table 2.

The numerical parameters are listed in table 3 . We use periodic boundary conditions in the three directions and the cases with non-zero Galileo numbers have a computational domain elongated in the vertical direction in order to reduce the effect of periodicity in the presence of vertically-elongated structures; this point will be further discussed in section 3 . For those cases we used a discretization at the particle scale $D / \Delta x=24$ in accordance to the former work of Uhlmann and Dušek [57] and Uhlmann and Doychev [56], while the case without gravity uses $D / \Delta x=16[55]$.

The particles of the cases G178, G180-R140, and G0-R120 are initially randomly distributed. We tested the resistance of the clusters formed in G178 to turbulence by using the final state of G178 as an initial state for G178-R95. Figure 1 gives a visual impression of both types of initial positions (columnar vs random). In G178-R95 the system starts with developed columns of particles and the associated particle-induced turbulence, then the turbulence is activated. In a single phase case, turbulence is known to become homogeneous isotropic after a transient phase of typically 3 large-eddy time scales $[7,13]$ and we ensured our computations to have an observation time larger than $8 \tau_{e}$. In the case G180-R140 we proceeded conversely: turbulence is forced until 


\begin{tabular}{|c|c|c|c|c|c|c|c|c|c|c|}
\hline Name & $N_{p}$ & $N_{x} \times N_{y} \times N_{z}$ & $\mathcal{L}_{z} / \mathcal{L}_{x}$ & $L^{S P} / \mathcal{L}_{x}$ & $\eta^{S P} / \Delta x$ & $\mathcal{L}_{x} / D$ & $D / \Delta x$ & $T_{o b s} / \tau_{e}^{S P}$ & $T_{o b s} / \tau_{p}$ & $T_{o b s} / \tau_{g}$ \\
\hline G178-R95 & 11867 & $2048 \times 2048 \times 4096$ & 2 & 0.39 & 3.57 & 85 & 24 & 8.7 & 53.28 & 837 \\
\hline G180-R140 & 11868 & $2048 \times 2048 \times 4096$ & 2 & 0.56 & 2.82 & 85 & 24 & 14.9 & 90.64 & 1350 \\
\hline G178 & 11867 & $2048 \times 2048 \times 4096$ & 2 & - & - & 85 & 24 & - & 98.98 & 1472 \\
\hline G0-R120 & 20026 & $2048 \times 2048 \times 2048$ & 1 & 0.43 & 2.89 & 128 & 16 & 25.0 & 460.64 & - \\
\hline R95 & 0 & $1024 \times 1024 \times 1024$ & 1 & 0.39 & 3.57 & - & - & 28.5 & - & - \\
\hline R140 & 0 & $512 \times 512 \times 512$ & 1 & 0.56 & 0.704 & - & - & 80.5 & - & - \\
\hline $\mathrm{R} 120$ & 0 & $512 \times 512 \times 512$ & 1 & 0.43 & 0.73 & - & - & 24.3 & - & - \\
\hline
\end{tabular}

Table 3: Main numerical parameters used for the present simulations: Number of particles $N_{p}$, extent $\mathcal{L}_{i}$ of the domain in the direction $i$ and corresponding number of Eulerian grid points $N_{i}$, elongation of the domain in the vertical direction $\mathcal{L}_{z} / \mathcal{L}_{x}$, ratio between the single phase large-eddy length scale and box size $L^{S P} / \mathcal{L}_{x}$ and between the single phase Kolmogorov length scale and grid width $\eta^{S P} / \Delta x$, observation time $T_{o b s}$ normalized by the single phase large-eddy time scale $\tau_{e}^{S P}$, particle time scale $\tau_{p}=D^{2} \rho_{p} /\left(18 v \rho_{f}\right)$ and gravitational time scale $\tau_{g}=D / u_{g}$. For all cases the length of the domain in both horizontal directions are set to be equal $\left(\mathcal{L}_{x}=\mathcal{L}_{y}\right)$.

statistically steady state is reached, then we add the particle and keep them fixed with respect to the computational grid while adding a constant positive vertical velocity $w_{s h}$ to the initial flow. During this phase the space resolution is low $D / \Delta x=12$, then we increase the resolution to $D / \Delta x=24$ by linear interpolation and release the particles. For both cases G178-R95 and G180R140 the turbulence forcing is adapted to account for this shift in the vertical component of the velocity [7]. The cases with $2048^{2} \times 4096$ grid points required roughly 10 million core-hours each on 8000 processor cores at LRZ Munich, Germany. Apart from differing in the initial condition, the cases G178-R95 and G180-R140 also differ by the Reynolds number of the forced turbulence, and, as a consequence, the resulting turbulence intensity $u_{r m s} / V_{T}$ differs from 0.14 to 0.22 , and $D / \eta$ is slightly different (6.8 vs 8.5 cf. tables 1 and 4 ).

\section{Description of the fluid phase}

We discuss in this section the behaviour of the fluid phase, focusing first on the evolution of the energy budget and the modification of the scales of the background flow, and then moving on to the description of the coherent flow structures.

\subsection{Influence of the particles on the background flow}

We propose to focus first on the energy budget in order to discuss how particles affect the global scales of the flow. For this we introduce the instantaneous kinetic energy $E_{k}(t)=\mathbf{u} \cdot \mathbf{u} / 2$. Its transport equation can be derived from the momentum equation 1 and then integrated over the entire domain $\Omega$ (occupied by both fluid and particles) with the space averaging operator $\langle.\rangle_{\Omega}$ [7]. This leads to:

$$
\frac{\mathrm{d}\left\langle E_{k}\right\rangle_{\Omega}}{\mathrm{d} t}=-\varepsilon_{\Omega}+\psi^{(t)}+\psi^{(p)}
$$


where $\varepsilon_{\Omega}(t)=2 v\left\langle S_{i j}^{\prime} S_{i j}^{\prime}\right\rangle_{\Omega}$ is the instantaneous box-averaged dissipation, $\psi^{(t)}(t)=\left\langle\mathbf{u} \cdot \mathbf{f}^{(t)}\right\rangle$ the work done by the turbulence forcing and $\psi^{(p)}$ the fluid-particle coupling term (eq. 8).

$$
\psi^{(p)}(t)=\left\langle\mathbf{u} \cdot \mathbf{f}^{(i b m)}\right\rangle_{\Omega}-\langle\mathbf{u}\rangle_{\Omega} \cdot\left\langle\mathbf{f}^{(i b m)}\right\rangle_{\Omega}
$$

Figure 2 gives the time evolution of the different terms in the budget of the volume averaged kinetic energy, scaled by the equivalent single phase dissipation $\varepsilon^{S P}$. Our previous works $[7,55]$ showed that in the absence of gravity, and for $D / \eta$ and $\Phi_{s}$ of similar range as the values explored in the current paper, the budget is barely affected by the presence of the particles and the system can be consequently seen as macroscopically one-way coupled. For a given non-zero Galileo number we observe here that the energy budget, and more specifically the dissipation, gets more affected while decreasing $D / \eta$. This can be emphasized by developing the fluid-particle forcing term into three contributions [7]:

$$
\psi^{(p)}(t)=\phi_{s}\left(\frac{\rho_{p}}{\rho_{f}}-1\right) \mathbf{u}_{r e l, \Omega} \cdot \mathbf{g}+\psi_{\text {accel }}^{(p)}(t)+\psi_{\text {coll }}^{(p)}(t),
$$

with the apparent slip velocity $\mathbf{u}_{r e l, \Omega}=\left\langle\mathbf{v}^{(i)}\right\rangle_{p}-\langle\mathbf{u}\rangle_{\Omega}$ (where the operator $\langle.\rangle_{p}$ refers to the average over the particles), $\psi_{\text {accel }}^{(p)}$ the contribution due to particle acceleration and $\psi_{\text {coll }}^{(p)}$ the contribution due to inter-particle collisions. The reader is referred to the appendix of Chouippe and Uhlmann [7] for more details on the derivation of eq. 9. In the dilute regime, the term $\psi_{\text {accel }}^{(p)}(t)+\psi_{\text {coll }}^{(p)}(t)$ is negligible compared to $\psi_{\text {pot }}^{(p)}(t)=\phi_{s}\left(\frac{\rho_{p}}{\rho_{f}}-1\right) \mathbf{u}_{r e l, \Omega} \cdot \mathbf{g}$, which can be normalized according to

$$
\frac{\psi_{\text {pot }}^{(p)}}{\varepsilon^{S P}}=\phi_{s} G a^{3}\left(\frac{D}{\eta^{S P}}\right)^{-4} \frac{w_{r e l, \Omega}}{u_{g}}
$$

Interestingly, if we propose to estimate, as a first level of approximation, the relative velocity $w_{\text {rel }} \approx V_{T}^{S N}$ with $V_{T}^{S N}$ the terminal velocity of one single particle estimated according to the drag law of Schiller and Naumann [47] it turns to give $\psi^{(p)} / \psi_{\text {pot }}^{S N} \sim 0.94(0.92)$ for G178-R95 (G180$\mathrm{R} 140)$. As $\phi_{s}, G a, V_{T}^{S N} / u_{g}$ have the same order of magnitude for both cases, this shows the importance of $D / \eta^{S P}$ on the enhancement of the dissipation level. The estimate that the average settling velocity is equal to the terminal settling velocity of a single particle in ambient fluid $\left(w_{\text {rel }} \approx V_{T}^{S N}\right)$ is reasonable; below we will find that for relative turbulence intensities around 0.2 deviations of the order of one percent are observed, while in the absence of background turbulence the difference amounts to 12 percent (cf. section 4.1).

Once averaged over the domain filled by the fluid we get a ratio for the two-phase dissipation $\varepsilon_{\Omega_{f}}^{T P} / \varepsilon^{S P}$ of the order of 18 for G178-R95 and 6.4 for G180-R140. We observe that the turbulence forcing power input is barely affected by the presence of the particles meaning that the main discrepancy is carried by $\psi^{(p)}$. We introduce a surrogate dissipation $\tilde{\varepsilon}^{S P}=\varepsilon_{\Omega}^{T P}-\psi^{(p)}$, and observe that it equals $0.88 \varepsilon^{S P}$ for G178-R95 and $1.02 \varepsilon^{S P}$ for G180-R140. As a first level of approximation the increase of the dissipation to be observed on figure 7 would follow $\varepsilon^{S P} \sim \varepsilon_{\Omega}^{T P}-\psi_{\text {pot }}^{S N}$ which attributes this increase to the release of potential energy of the particles during the settling, in accordance with the former observations of Chouippe and Uhlmann [7] and Hwang and Eaton [30].

Figure 3 gives the evolution of the autocorrelation of the fluid velocity fluctuations in the z-coordinate direction. In Chouippe and Uhlmann [7] we have seen that the single phase configuration with elongated domains in the vertical direction follows its cubic equivalent up to distances equal to the maximal separation in the direction $\mathrm{x}\left(\mathcal{L}_{x} / 2\right)$ where we observe almost no 


\begin{tabular}{rccccccc}
\hline Name & $u_{r m s}^{S P} / V_{T}$ & $u_{r m s}^{T P} / V_{T}$ & $u_{r m s, h o r}^{T P} / V_{T}$ & $u_{r m s, v e r}^{T P} / V_{T}$ & $V_{T}^{\text {turb }} / V_{T}$ & $v_{r m s, h o r} / V_{T}$ & $v_{r m s, v e r} / V_{T}$ \\
\hline G178-R95 & 0.14 & 0.16 & 0.14 & 0.20 & 1.011 & 0.19 & 0.18 \\
G180-R140 & 0.22 & 0.23 & 0.22 & 0.25 & 0.994 & 0.30 & 0.22 \\
G178 & - & 0.17 & 0.09 & 0.27 & 1.12 & 0.08 & 0.26 \\
\hline
\end{tabular}

Table 4: Scales of the fluid velocity $\mathbf{u}$ and the particle velocity $\mathbf{v}$, scaled by the settling velocity of one particle falling in quiescent fluid $V_{T}$. The velocity $V_{T}^{\text {turb }}$ corresponds to the terminal velocity for multiparticle settling in forced turbulence $\left\langle\left|w_{\text {rel }}\right|\right\rangle_{t}$.

correlation, then the autocorrelation follows a monotonic increase until the maximal separation. This behavior is to be interpreted as a signature of the turbulence forcing that injects energy for every two modes in the z-direction, and is conserved in the turbulent particle-laden cases: $\mathcal{R}_{w w}$ first decreases until $\mathcal{L}_{x} / 2$ where the correlation is weak (this point is consistent with the single phase case R95), and then increases monotonically until the maximal separation where we observe that the flow is not fully decorrelated. The successive decrease and increase can hence be attributed to the forcing scheme, but the amount of correlation at the maximal separation is also a consequence of the wakes influence. Indeed in the pure multiparticle case G178 we observe that the flow does not fully decorrelate [56]. The level of correlation at $\mathcal{L}_{x} / 2$ also monotonically increases with $u_{v e r, r m s}^{T P} / u_{g}$, which is counter-intuitive as one would expect turbulence to decrease the influence of the wakes. This trend can still be seen as a signature of particles, and more specifically to the formation of clusters, which will be discussed in the next section, since we observe that the level of autocorrelation actually increases with the clustering level. Uhlmann and Doychev [56] have suggested to increase the domain size in the vertical direction to decrease this correlation, but they also estimated that even an elongation of $\mathcal{L}_{z} / \mathcal{L}_{x}=8$ would be insufficient to resolve this issue.

We now turn to the influence of the forced turbulence and the wakes on the scales of the flow velocity. Two contributions are here in competition as noted by previous authors $[7,30$, 51]: on the one hand the increase of the dissipation due to the gradients at the fluid-particle interface should tend to decrease the level of turbulent kinetic energy, but on the other hand the development of the wakes should induce the formation of small scale structures which increases velocity fluctuations. Table 4 gives the values for the ratios between the fluctuating velocities and settling velocity for both fluid and particle phases. We first observe that particles at the current Galileo numbers tend to globally enhance the fluctuating velocity as $u_{r m s}^{T P}$ is larger than $u_{r m s}^{S P}$ for both G178-R95 and G180-R140 cases. We choose to split the amount of fluctuations into its horizontal and vertical fluctuations, respectively $u_{r m s, h o r}^{T P}=\left[0.5\left(\left(u_{x}^{\prime}\right)^{2}+\left(u_{y}^{\prime}\right)^{2}\right)\right]^{1 / 2}$ and $u_{r m s, v e r}^{T P}=\left(\left(u_{z}^{\prime}\right)^{2}\right)^{1 / 2}$. We observe that the horizontal contribution is of the same order as its single phase equivalent, meaning that the wakes mostly affect the vertical component of velocity fluctuations. Besides the turbulent cases feature lower anisotropy levels than in the pure multiparticle settling, with a ratio $u_{r m s, v e r}^{T P} / u_{r m s, h o r}^{T P} \approx 1.61$ for G178-R95 and 1.19 for G180-R140, while $u_{r m s, v e r}^{T P} / u_{r m s, h o r}^{T P} \approx 9.90$ for the original Ga178 configuration.

Despite this clear anisotropy of the flow we use the standard definitions of the Kolmogorov length scale, Taylor micro scale, fluctuating velocity and associated Reynolds number. The increase of the dissipation and the formation of small scale structures in the wakes leads on one side to an increase of the Kolmogorov length scale $\eta^{T P}$, but on the other side hardly affects the level of turbulent kinetic energy. This point can be partially attributed to the fact that the level of fluctuations induced by the particles $u_{r m s}^{T P}$ of the pure settling case G178 is actually of the 
same order as the level of fluctuations $u_{r m s}^{S P}$ used to force turbulence in the case G178-R95. The amount of particle-induced turbulence is therefore probably not strong enough to induce by itself larger modifications of the fluctuations amplitude. We also introduced two Taylor micro scale deduced from the transverse autocorrelation functions, namely $\lambda_{\text {hor }}$ and $\lambda_{\text {ver }}$ respectively related to $\mathcal{R}_{u u}\left(r_{z}\right)$ and $\mathcal{R}_{w w}\left(r_{x}\right)$. Based on this we introduce two separate Reynolds numbers to account for the anisotropy, namely:

$$
\begin{aligned}
\operatorname{Re}_{\lambda, h o r}^{T P} & =\frac{\lambda_{\text {hor }} u_{r m s, h o r}^{T P}}{v} \\
\operatorname{Re}_{\lambda, v e r}^{T P} & =\frac{\lambda_{\text {ver }} u_{r m s, v e r}^{T P}}{v} .
\end{aligned}
$$

The resulting Reynolds numbers are listed in table 5.

We observe that the Taylor microscale in the horizontal direction are closer to the single phase

\begin{tabular}{rcc}
\hline Name & $R e_{\lambda, \text { hor }}^{T P}$ & $\operatorname{Re}_{\lambda, \text { ver }}^{T P}$ \\
\hline G178-R95 & 90.00 & 63.03 \\
G180-R140 & 168.52 & 107.50 \\
G178 & 31.64 & 108.55 \\
\hline
\end{tabular}

Table 5: Two-Phase Reynolds numbers computed according to equations 11 and 12 for which the Taylor microscale has been deduced from the transverse two-point correlations. The distinction is also made for the characteristic fluctuating velocities in the horizontal and vertical directions.

value while it is closer in the vertical direction to the pure multiparticle settling configuration, resulting in an increase of the Taylor microscale in the horizontal direction and a decrease in the vertical direction. We observed that the presence of the particles barely affects the amplitude of the horizontal fluctuations and tend to increase this amplitude in the vertical direction. This explains that the presence of the particles tends to increase the Reynolds numbers in the horizontal direction and decreases them in the vertical direction.

\subsection{Large and small scale structures of the flow}

We now consider the turbulent structures, and propose for this to base our analysis on the $Q$ criterion proposed by Hunt et al. [29], and defined as $Q=u_{i, i} u_{i, i} / 2-u_{i, j} u_{j, i} / 2$. Figure 4 gives the probability distribution of $Q$; the figure also states the values of the corresponding standard deviations scaled by $\omega_{r m s}$ (defined as $\omega_{r m s}=\sqrt{\varepsilon / v}$ ). It shows that turbulence tends to increase the amplitude of the peak of $Q$ compared to the mutliparticle settling case G178. The presence of the particles increases the standard deviation of $Q$ due to the enhancement of the velocity gradients at the surface of the particles. We represent the coherent structures by isocontours of $Q$ taken at a given threshold of the standard deviation (blue contours on figures 5 and 6). We chose as threshold $1.5 \sigma(Q)$ in the absence of gravity and $0.5 \sigma(Q)$ for the settling cases. The adjustment of the threshold for the settling cases was motivated by the choice to stay at a roughly equal probability to meet the corresponding $Q$ among the cases (i.e. the depicted isosurfaces roughly enclose the same total volumes in figures 5 and 6). The visualizations feature the well known elongated worm-like vortices for both single phase and particle-laden configurations (figures 5 and 6). Uhlmann and Chouippe [55] have shown the presence of wakes (with volumes below the particle volume) in the vicinity of the particles for $G a=0$. In the settling 
cases, the small-scale structures are on contrary mainly dominated by the wakes arising from the mean settling, and which lead to the increase of the standard deviation of $Q$. This does not mean that worm-like structures are completely destroyed by the wakes however, but that they are not the dominant small scale vortical structures at $0.5 \sigma(Q)$ (those worms are for example still present in the visualization of G180-R140 which features the largest turbulence intensity). We recall that, in the absence of forced turbulence, the large-scale structure of the flow is characterized by large columns of wakes, that are here clearly visible (fig. 5). For the forced cases those large patterns are less evident though, and we filter the velocity field with a filter width $\Delta_{\text {filt }}$ with the aim of extracting more clearly those large eddies. We took a filter width $\Delta_{\text {filt }}=3.7 \mathrm{D}$ for G0-R120, and $\Delta_{\text {filt }}=5.6 \mathrm{D}$ for the other cases (we used the same width in multiple of the Kolmogorov length in the single-phase case R95). As expected, filtering smoothes out the velocity gradients, decreases the standard deviation of $Q$ and lets its pdf tend towards Gaussian (cf. figure 4). We used this criterion to visualize the large-scale structures of the flow in the same manner as for the unfiltered field, this time with the threshold $1.5 \sigma\left(Q_{\text {filt }}\right)$ for all cases (where $Q_{\text {filt }}$ is the box-filtered $Q$ field). No adjustment of the threshold was here necessary for the settling cases to ensure that the corresponding probabilities of $Q_{\text {filt }}$ stay roughly equal, and that this thresholds provided similar probability to its unfiltered equivalent (yellow contours on figures 5 and 6). The large scale structure obtained with the filtering procedure features similar characteristics as those obtained by representation of the low pressure regions of the unfiltered field. In the absence of gravity both single phase and particle-laden cases feature elongated vortices of larger size in the vicinity of the smaller worms that concentrate inside and around those large eddies. Note that this "vortex-within-vortex" scenario has been documented in previous singlephase studies (cf. e.g. the excellent visualization of Bürger et al. [6]). In the pure sedimentation case, we observe the presence of large structures that most probably result from the formation of vortical structures in the mixing zones induced by the wake columns, in accordance with the observation of Uhlmann and Doychev [56]. As expected, forced turbulence reduces the signature of those vertical columns that become barely visible on the visualizations (fig 6). It also seems that large-scale structures of similar size as the single phase equivalent are still present, with an orientation mostly directed in the vertical and horizontal direction. This behaviour is consistent with the description of the "banana-shape" structures proposed by Ferrante and Elghobashi [15], but a more systematic analysis should be performed to measure the impact of the settling on the large eddies of the flow.

Cases G178-R95 and G180-R140 differ mainly by the interaction between large vortices and the wakes, and consequently the concentration of small-scale vortices with respect to the larger ones. On the one hand the wakes mostly disrupt and shorten the large-scale vortical structures for the case G178-R95, while on the other hand they also accumulate in the vicinity of the large eddies for the case G180-R140. As a result, relatively large regions with a more important density of wakes develop for G180-R140, while for G178-R95 those regions have a smaller extent and do not seem to sample preferential regions of the filtered field. This trend is consistent with the level of concentration that will be described in the next section. G178-R95 and G180-R140 do not only differ by their turbulence intensity, but also by the length scales of the flow with respect to the particle size. The Kolmogorov length is slightly smaller for Ga180-R140 $\left(\eta^{S P}=0.12 D\right)$ than for Ga178-R95 $\left(\eta \eta^{S P}=0.15 D\right)$ while large-eddy length are larger for Ga180-R140 $\left(L^{S P}=47.8 D\right)$ than for Ga178-R95 $\left(L^{S P}=32.8 D\right)$. As an effect, one could expect the particles and their wakes to be more affected by the large scales of the forced turbulence for the case G180-R140 than G178R95, by getting expelled outside of those large scale vortices according to the model initially proposed for point-particles. This could explain the observation on figure 6 that, contrarily to the case G178-R95, some particles seem to be located in the vicinity of the large structures in the case G180-R140 and probably rotating around them according to the orientation of their wakes. 
A more systematic investigation of those events (including an analysis of time-resolved data) is however necessary to conclude on their statistical evidence as well as their actual impact on potential particle accumulation.

\section{The dispersed phase}

We now the behaviour of the dispersed phase and propose to focus first on the possible modification of the settling velocity of the particles and considering then the ability of the particles to form large or small scale clusters.

\subsection{Settling velocity}

The interaction with the background turbulence will also lead to a modification of the settling velocity of the particles. To estimate this settling velocity we need to define a relative velocity between the particles and the fluid. To do so we first use an apparent velocity $\mathbf{u}_{r e l}^{(i)}=\left(u_{r e l, x}^{(i)}, u_{r e l, y}^{(i)}, u_{r e l, z}^{(i)}\right)^{T}$ corresponding to the difference between the ith particle velocity and the mean flow, viz.

$$
\mathbf{u}_{r e l}^{(i)}(t)=\mathbf{v}^{(i)}(t)-\langle\mathbf{u}\rangle_{\Omega_{f}}(t) .
$$

We introduce then the average settling velocity $w_{\text {rel }}$ corresponding to the average over all particles of the vertical component of this apparent slip velocity, namely

$$
w_{r e l}(t)=\left\langle u_{r e l, z}^{(i)}\right\rangle_{p}(t)
$$

Figure 7 gives the time evolution of this velocity, scaled by the gravitational velocity scale $u_{g}$. It shows that turbulence tends to decrease the settling velocity for both cases G178-R95 and G180R140 with respect to the multiple particle settling configuration G178, in accordance with the observations of Fornari et al. [17] and Chouippe and Uhlmann [7]. It shows that the resulting settling velocity tends towards the value of one single particle falling in quiescent flow, with a difference of few percents. Case G178-R95 features an increase of 1.1\% while G180-R140 shows a decrease of $0.6 \%$. Nonlinear drag effects are known to induce a reduction of the settling velocity $[41,52,58]$, and Homann et al. [27] proposed a model to estimate the amplitude of this reduction depending on the turbulence intensity. This model relies on adding a Gaussian perturbation that possesses the same standard deviation as the forced turbulence to the velocity seen by the particles, and to estimate how the induced modification of the drag would affect the mean settling velocity. We applied this model to the configurations explored here and found that turbulence should induce a reduction of the settling velocity of $0.7 \%$ (3.1\%) for case G178-R95 (G180-R140). This model is not meant to reproduce collective effects (e.g. wake attractions, modification of the flow structures), but partially explains the reductions observed here.

In order to account for the local properties of the velocity field in the vicinity of the particles, we introduce for each particle with index " $i$ " a velocity $\mathbf{u}^{\mathcal{S}_{(i)}}$ defined as the average velocity over a sphere $\mathcal{S}_{(i)}$ of radius $R_{S}$ and centered at the particle's center. We then define the corresponding relative velocity $\mathbf{u}_{r e l} \mathcal{S}_{(i)}$, according to

$$
\mathbf{u}_{r e l}^{\mathcal{S}_{(i)}}(t)=\mathbf{v}^{(i)}(t)-\mathbf{u}^{\mathcal{S}_{(i)}}(t) .
$$

This definition has been introduced in [35] and tested for different shell radii $R_{S}$. Using a radius $R_{S}=1.5 D$ has been shown to be a good compromise between not being too much affected by the particle wakes and still being representative of the local properties of the surrounding velocity 
field. We introduce a subsequent settling velocity $w_{\text {ref }}^{\mathcal{S}}$ defined as the vertical component of the particle average relative velocity based upon sphere-averaging, viz. $w_{r e f}^{\mathcal{S}}=\left\langle u_{r e l, z}^{\mathcal{S}_{(i)}}\right\rangle_{p}$. The time evolution of this settling velocity is also represented on figure 7. Similar to the pure multiparticle settling case, it shows that the Reynolds number based upon the sphere-averaged velocity (eq. 16) is always smaller than its global equivalent, meaning that the particle velocity tends to adapt to its surrounding. Here as well a monotonous trend is observed with respect to the influence of the relative intensity of the turbulence, with a smaller falling velocity obtained for the largest turbulence intensity. It appears indeed that the turbulence intensity tends to monotonously affect the mean apparent relative velocity $w_{r e l}$ as well as the mean vertical component representative of the velocity difference between the particles and their vicinity $w_{\text {rel }}^{\mathcal{S}}$ (cf. figure 7).

We introduce the instantaneous angle $\alpha_{p}$ between the relative particle velocity vector and the vertical axis, defined by the following relation

$$
\tan \left(\alpha_{p}\right)=\frac{\sqrt{u_{r e l, x}^{2}+u_{r e l, y}^{2}}}{\left|u_{r e l, z}\right|} .
$$

The p.d.f. of the settling angle is depicted on figure 8 and shows a shift of the peak of probability towards larger angles with increasing turbulence intensity, as well as a broadening of the distributions. Note that the peak of probability for the case G178 is approximately located at the angle which would be observed in the case of one isolated sphere settling in a still fluid (i.e. $\alpha_{p}=5.2^{\circ}$ ). We introduce three reference angles $\tan \left(\alpha_{r e f}^{i}\right)=\left(2 u_{r m s}^{S P}\right)^{1 / 2} /\left|V_{T}+i \times u_{r m s}^{S P}\right|$ with $i=\{-1,0,1\}$, and observe that the peak is centered around the reference angle $\alpha_{\text {ref }}^{0}$. The spreading of the distribution seems also to be linked with the enlargement of the range $\left[\alpha_{r e f}^{-1}, \alpha_{r e f}^{1}\right]$ with the turbulence intensity, which implies an increase of the relative horizontal motion of the particles induced by turbulence.

Figure 7(b) shows the normalized p.d.f. of the resulting Reynolds number (based on the sphere-averaged relative velocity). Interestingly, the shape of the distribution does not highlight any significant influence of the forced turbulence suggesting that the evolution of the local motion of the particle is dominated by the wakes. This last point can also be emphasized by considering the p.d.f. of the fluid velocity (not the relative velocity), represented on figure 9 . The distinction has been indeed made between p.d.f. accounting for the total fluid domain, and the domain occupied by the spheres $\mathcal{S}_{(i)}$. It shows a mild increase of the probability for finding large vertical and horizontal velocities in the vicinity of the particles. These points will be further discussed in $\S 5.2$. We then slightly adapted the definition of the local velocity $\mathbf{u}^{\mathcal{S}_{(i)}}$ such as to account only for the flow upstream of the particles. To do so we first introduce an "incoming" flow velocity $\mathbf{U}_{\text {in }}^{(i)}$ taken as the negative particle velocity, viz.

$$
\mathbf{U}_{i n, 1}^{(i)}(t)=-\mathbf{v}^{(i)},
$$

or as the negative sphere-averaged relative velocity similar to the work of Cisse et al. [9]:

$$
\mathbf{U}_{i n, 2}^{(i)}(t)=-\mathbf{u}_{r e l}^{\mathcal{S}_{(i)}}(t) .
$$

Next we average over the set of points $\mathbf{x}$ located upstream of the particle, i.e. those for which the following condition holds:

$$
\left(\mathbf{x}-\mathbf{x}_{\mathbf{p}}{ }^{(i)}\right) \cdot \mathbf{U}_{i n, j}^{i}<0 \text { with } j=1,2 .
$$

In the following we will denote the relative velocity obtained with the first and second definitions by $\mathbf{u}_{r e l, f 1}^{\mathcal{S}_{(i)}}$ and $\mathbf{u}_{r e l, f 2} \mathcal{S}_{(i)}$ respectively, the corresponding fluid velocity sampled at the front of 


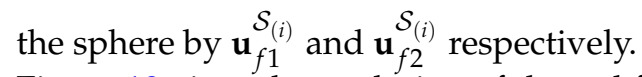

Figure 10 gives the evolution of the p.d.f. of the fluid sampled either on the totality of the fluid domain, or on the totality of the particle-centered sphere $\mathcal{S}_{(i)}$ with a radius equal to three times the particle radius, or on the front of the spheres according to both definitions. As we can see the distinction made on the portion of the spheres to be sampled for the statistics has little influence. This distinction has a slightly larger affect on the resulting relative velocity between the particles and the incoming fluid (figure 7). The absolute value of the settling velocity obtained with the two variants where the fluid velocity seen by the particles is computed on the upstream-facing, particle-centered hemisphere is systematically smaller by one to two percent. This result is presumably related to the typical structure of the wake flow in the vicinity of the particles which does not only feature a (low-velocity) downstream recirculation region, but also a (high-velocity) transverse displacement region. This point probably merits further investigation in the future. For the present purpose, however, the comparison of the three different definitions of a relative velocity confirm the observed mild trend of a decrease (in absolute value) with turbulence intensity of its mean value.

\subsection{Clustering}

We now turn to the tendency of the particles to form clusters. We choose to base our characterization of the clustering on the Voronoï tesselation analysis proposed by Monchaux et al. [39] and Monchaux et al. [40]. It consists in dividing the total volume into $N_{p}$ subvolumes $\mathcal{V}_{V o r}^{(i)}$ defined for each particle (i), such that every point contained in the volume $\mathcal{V}_{V o r}^{(i)}$ is closer to the ith particle's center than to any other particle. The volumes obtained can then be interpreted as the inverse of a concentration since dense regions containing more particles than the rest will be characterized by small Voronoï volumes and conversely. This method has several advantages: it provides an objective diagnostic for clustering by comparing the p.d.f. of the Voronoï volumes normalized to its random equivalent. A set or randomly distributed particles, with positions taken from random Poisson process will give a p.d.f. following a gamma distribution. This p.d.f. is universal with coefficients numerically estimated by Ferenc and Neda [14], but in the case of finite-size particle the p.d.f. looses its universality as it will be affected by the condition on particles to not overlap. The coefficients are then function of the particle volume fraction and the size of the total domain. The comparison between the p.d.f.'s of the cases considered here and the random equivalent represented on figure 12 is a typical example for clustering diagnostic: we indeed observe that the p.d.f.'s differ for all simulated cases to the random equivalent, in favor of an increase of probability to see small and large Voronoï volumes. The increase of the probability to have small Voronoï volumes is indeed to be interpreted as the sign of local enhancement of particle concentration (i.e. clusters) while the increase of the probability to have large volumes is the sign of local decrease of this concentration (i.e. voids). The two volumes $\left(\mathcal{V}_{V o r}^{c l u s}\right.$ and $\left.\mathcal{V}_{\text {Vor }}^{\text {void }}\right)$ corresponding to the crossing points between the p.d.f.s of the simulated cases and their random equivalent can then be taken as an objective criterion for determining whether particles belong to a cluster or a void region: every particle with a Voronoï volumes smaller than $\mathcal{V}_{V o r}^{\text {clus }}$ will belong to a cluster while every particle with a volume larger than $\mathcal{V}_{V o r}^{\text {void }}$ will belong to a void region [39].

We quantify the level of clustering with the evolution of the standard deviation of the Voronoï volumes. Its time evolution is represented on figure 11(a) and compared to the random configuration. A set of randomly distributed particles is characterized by a standard deviation approximately 0.4 while clustering is be characterized by an increase of this standard deviation. Figure 11 recalls that the pure multiparticle settling case G178 is characterized by significant clustering [56], and that the non-settling case G0-R120 with turbulence shows small but statistically significant 
clustering [55]. Here we observe in the case G178-R95 that turbulence tends to rapidly disturb the clusters present in the initial condition without completely destroying them, the resulting standard deviation of the Voronoï volume being larger than its random equivalent with an increase of roughly $10 \%$. Interestingly the influence of the turbulence intensity is non-monotonous (fig 11(b)), as the level of clustering is larger for G180-R140 than G178-R95. Both of them are indeed lower than in the pure multiparticle settling, meaning that the disturbance induced by the forced turbulence tends to decrease the level of clustering. But an increase of the turbulence intensity from G178-R95 to G180-R140 does not induce a decrease of the level of clustering. To our knowledge the work of Fiabane et al. [16] is the only one that details experimental evidence of clustering of finite-size for heavy particles in forced turbulence. The Galileo numbers investigated were of the order of 10, and the configurations studied evidenced strong clustering. However, the relative turbulence intensity in those experiments was much higher than here with $u_{r m s} / V_{T}$ ranging from 0.7 to 4.5 .

Let us now move on to the characteristics of the clusters, which are reconstructed as follows: we first extract the Voronoï volumes below the threshold $\mathcal{V}_{\text {Vor }}^{\text {clus }}$ and reconstruct the cluster by finding all cells that are connected. The simulations employ periodic boundary conditions in the three spatial directions and we included this periodicity in the reconstruction of the clusters. We slightly adapted the choice of the threshold $\mathcal{V}_{\text {Vor }}^{\text {clus }}$ in order to take a value common for all the cases. We indeed observed that this threshold has an impact on the volume $\mathcal{V}_{\text {clus }} /\left\langle\mathcal{V}_{\text {Vor }}\right\rangle$ featuring the highest probability. We selected $\mathcal{V}_{\text {Vor }}^{\text {clus }}=0.64\left\langle\mathcal{V}_{\text {Vor }}\right\rangle$, which is representative of the crossing points obtained for the cases simulated here (fig. 12). We obtained that the mean cluster volume $\left\langle\mathcal{V}_{\text {clus }}\right\rangle$ approximately equals to $235 \eta^{3}$ for G178-R95 and $3000 \eta^{3}$ for G180-140, corresponding to a linear dimension of the clusters of $6.2 \eta$ and $14.6 \eta$ respectively, which is of the same order as the previous observations of the literature [1, 42]. Figure 13 depicts the p.d.f.s of the obtained cluster volumes scaled by the mean Voronoï volume, for the cases simulated here as well as a set of random distributed particles which possesses the same geometrical properties (particle radius and size of the domain) and solid volume fraction as cases G178-R95 and G180-R140. It presents the same features as the p.d.f.'s observed with two-dimensional experimental visualizations by Sumbekova et al. [50] and in three-dimensional visualizations by Uhlmann and Chouippe [55], with a peak of cluster volume followed by an power law decay over nearly two decades and an exponential decay for the largest clusters.

Goto and Vassilicos [23] proposed a model distribution of clusters and voids for two-dimensional simulations by assuming that they mimic the self-similarity of turbulent eddies. By doing so they obtained an exponential decay of clusters area of $-5 / 3$, which have been further observed experimentally by Sumbekova et al. [50]. An extension of this model to three-dimensional cluster detection gives a -16/9 exponential decay [55], and provides a good trend of the decay for all the cases explored here. Interestingly the set of random particle positions considered here for comparison provides the same trend with a peak located between approximately $0.4\left\langle\mathcal{V}_{\text {Vor }}\right\rangle$ and $0.8\left\langle\mathcal{V}_{V o r}\right\rangle$, and an inertial range with exponential decay following the $-16 / 9$ slope. The difference to the randomness lies in the structure of the smallest and largest clusters that are more probable in the simulations, and in the extent of the inertial range. A possible scenario would be that turbulence tends to affect the smallest accumulation zones by making them smaller and therefore increasing the particle concentration. On the other side of the volume distribution we observe that turbulence increases the probability to observe large clusters compared to the random reference. The graph also shows that gravity likewise tends to increase the probability of finding large-volume clusters as compared to the random data, i.e. all cases involving particle settling are above the zero-gravity data for $\mathcal{V}_{c} /\left\langle\mathcal{V}_{V o r}\right\rangle \geq 200$. The observed differences between the settling case without turbulence (G178) and those with the two different levels of background turbulence (G178-R95, G180-R140) in terms of the cluster volume at the large-scale end of the spectrum are 
probably not significant as compared to the statistical uncertainty. However, we will see below that the clusters' geometrical aspect ratio is clearly affected by turbulence, which suggests that the principal action of turbulence is to modify the shape of gravity-induced clusters.

In order to get more insight into the geometrical properties of the clusters, we considered their spatial extension. To do so, we looked for the smallest cuboid, with faces aligned with the three global coordinate directions, that is able to contain each cluster, and we define the directional extension of the cluster as the side length of the corresponding cuboid. We then analyzed the aspect ratio, defined here as the ratio between the extension of the cluster in the vertical direction $L_{c}^{z}$ to its extension in any of the horizontal directions $L_{c}^{x}$ or $L_{c}^{y}$. The evolution of the mean aspect ratio of the clusters is shown in fig. 14. It shows that settling tends to elongate the largest clusters in the vertical direction. For any significant cluster size, the aspect ratio in general increases with the cluster volume. Here the largest values are obtained for the pure settling case G178, while in the forced turbulence cases G178-R95 and G180-R140 the elongation is significantly smaller. Interestingly, the curves corresponding to the two different turbulence intensities presently simulated practically collapse.

\section{Discussion}

We have seen in the previous sections that the relative intensity $I$ of the forced turbulence with respect to the original settling velocity does not necessarily bring a monotonous trend: although it progressively decreases the falling speed of the particles, it decreases the level of clustering without displaying a clear progression of this reduction with $I$. The visualizations based upon the $Q$ criterion highlighted a flow dominated at small scales either by worm-like vortical structures (in the absence of buoyancy) or by wakes (for $G a \neq 0$ ). The large scales are characterized by large vortices that are progressively disrupted by the wakes' contribution as turbulence intensity decreases. Here we will discuss several aspects of the interaction mechanisms suggested in the literature, focusing on the potential influence of the various time scales, on the centrifugal mechanism, and on the potentially related preferential sampling of the flow. A special attention will also be given regarding the tendency of gravity to increase particle accumulation compared to the situations featuring no buoyancy effect. As the large eddies of the flow appear to be affected by the presence of the particles, in the settling configurations, we discuss to which extent those two-way coupling effects can have an impact on the aforementioned interaction mechanisms.

\subsection{Considerations on the time scales}

It is common to introduce a Stokes number based upon the Kolmogorov time scale to measure the ratio between the time-scale of the particles and the typical time scale of the smallest eddies of the flow, namely $S t_{\eta}=\tau_{p} / \tau_{\eta}$, where the relaxation time scale of the particles $\tau_{p}=D^{2} \rho_{p} /\left(18 v \rho_{f}\right)$ is taken from the Stokes drag. Note that this definition of the particle time-scale is sometimes adapted in the literature in order to account for added mass effects, except if the density ratio is very large. In the case of point-particles it is commonly expected to see maximal preferential accumulation for Stokes number of the order of $1[3,10]$. Yoshimoto and Goto [62] have shown that clustering can still appear for larger Stokes number, meaning that not only the smallest eddies are playing a role in the clustering mechanism but larger ones can be involved. They introduced a resonance condition, namely that eddies of size $\ell$ and timescale $\tau(\ell)$ will centrifuge out a heavy particle with a relaxation time scale $\tau_{p}$ if the corresponding Stokes number $S t_{\ell}=\tau_{p} / \tau(\ell)$ is contained between 0.1 and 2. Preferential accumulation results then from the interaction with the range of eddies for which the resonance condition is fulfilled. 
In the absence of gravity, we observe here a relatively small degree of clustering for G0-R120, which is in accordance with the resonance model since $S t_{\eta} \approx 2.5$ and $S t_{e} \approx 0.05$ for this case. The flow should then contain eddies that are capable of expelling particles. The particle will barely feel the influence of any smaller eddies, and will be swept by the larger ones. The order of magnitude of $S t_{\eta}$ indicates however that the smallest scales verifying this resonance condition should be of the same order as the Kolomogorov length-scale, and consequently smaller than the particles themselves (we remind that this case features $D / \eta \approx 5.5$ ). The centrifugal scenario is hence not realistic at the small range, which might explain that the accumulation level achieved is actually low. Uhlmann and Chouippe [55] considered another configuration at $G a=0$ (cf. their case "D11") which features the same turbulence characteristics as the current G180-R140 case, with slightly larger particles $(D / \eta=11)$. This case features Stokes numbers $S t_{\eta}=10.7$ and $S t_{e}=0.29$, and, consistently, presents smaller accumulation level than G0-R120. Judging from the consideration of these two reference cases without gravity, it would therefore be expected from a configuration presenting the same geometrical and turbulence characteristics as G180R140 but no gravity effects to display a relatively small clustering level. This means that once gravity effects set in, we obtain a global enhancement of this particle accumulation, which can be potentially explained by different mechanisms that we will discuss next.

The first one is the attraction between particles, that is induced by their wakes and which is clearly at play in the pure settling case (G178). It would be then expected to see the level of clustering monotonously decreasing with the turbulence intensity, contrarily to the trend observed. Consequently this mechanism does not completely explain the enhancement of the accumulation.

Another candidate that can be proposed concerns the influence that gravity has on the interaction time scales between fluid and particles. In the original resonance model, the time-scale $\tau(\ell)$ of an eddy $\ell$ does not correspond to the time actually seen by a particle settling through this eddy, which can be roughly estimated by $\tau^{(S)}(\ell)=\ell / V_{T}$. This concept can be connected to the so-called "crossing trajectory effect" introduced by Yudine [63], which refers to the tendency of heavy particles to continuously change their fluid neighborhood while falling, resulting in a decorrelation of the fluid velocity along particles trajectories [43, 59]. Crossing trajectory effects appear to decrease particle dispersion and to have a negligible influence for turbulence intensity larger than unity [11,59]. It can be first verified that the settling time scale does not exceed its characteristic time scale with the condition $\tau(\ell)>\tau^{(S)}(\ell)$. This is indeed the case, since for the large eddies we have $\tau_{e} / \tau_{e}^{(S)}=1 / I$ (with $I<1$ ), and for the Kolmogorov length scale $\tau_{\eta} / \tau_{\eta}^{(S)} \sim \sqrt{R e_{\lambda}} / I$. We introduce the surrogate Stokes number $S t_{\ell}^{(S)}=\tau_{p} / \tau_{\ell}^{(S)}$, which takes into account the reduced interaction time due to the mean relative velocities. It shows that turbulence intensity tends to decrease the Stokes number based on the large scales according to $S t_{e}^{(S)}=S t_{e} / I$, and that both Reynolds number and relative turbulence intensity affect the Stokes number of the small scales with $S t_{\eta}^{(S)}=S t_{\eta} \sqrt{R e_{\lambda}} /\left(15^{1 / 4} I\right)$. Gravity will consequently have the effect of increasing the value of the apparent Stokes number representative of the largest eddies, which would tend to promote the occurrence of preferential accumulation. Gravity will also have the effect of filtering out the influence of the smallest eddies of the flow upon the particle motion by increasing $S t_{\eta}^{(S)}$.

In order to have a better impression on how this can affect the motion of the particle, we go back to the size $\ell$ of the eddy that would realize $\tau_{p} / \tau(\ell)=\alpha$. This eddy should verify

$$
\frac{\ell}{D}=\left(\frac{D}{\eta}\right)^{2}\left(\frac{\rho_{p}}{18 \alpha \rho_{f}}\right)^{2}
$$


This can be used to estimate the size of the smallest eddy realizing the resonance condition $(\alpha=$ $2)$, which in the present case turns out to yield non realistic values $(\ell / D \approx 0.4$ for G178-R95 and $\ell / D \approx 0.6$ for G180-R140). This means that this resonance can only be verified at larger scales, leading to a narrower range of resonance. Looking now at the size of the eddy $\ell$ that would verify the settling-aware condition on the time-scale ratio, viz. $\tau_{p} / \tau^{(S)}(\ell)=\alpha$ leads to the following relation for the eddy size:

$$
\frac{\ell}{D}=\frac{\rho_{p}}{18 \alpha \rho_{f}} R e_{T}
$$

with $\operatorname{Re}_{T}=D V_{T} / \nu$. In this context, the smallest eddies verifying the resonance condition $(\alpha=2)$ would be of the order of $10 \eta$. This means that the centrifuge mechanism leading to particle accumulation that these flow structures have upon the particle motion is potentially active.

\subsection{Preferential sampling}

We now turn to the structure of the velocity field. According to the preferential sweeping scenario of Wang and Maxey [58], the particles preferentially sample the downward-flowing regions, resulting in increased settling speed. Contrarily, in the present case we have seen that no clear modification of the settling speed is observed (section 3), with a small decrease of the velocity obtained for G180-R140 and a small increase for G178-R95. We have seen that non-linear drag effects could explain the slight speed reduction for the case G180-R140, but that by itself it would induce a slight reduction in the other one as well. We have therefore investigated whether particles sample specific regions of the flow to test if there is a potential competition between fast tracking and non-linear drag effects. We considered for this the probability density of the fluid velocity field, as represented on figure 9. We separated the horizontal and vertical components and observed a similar trend as what was obtained at lower Reynolds number [7] or in vertical turbulent channel flow [54], namely that the fluid velocity fluctuations lose their Gaussian behaviour for amplitudes larger than approximately three times their standard deviations, and that a negative skewness arises, due to the formation of wakes. Note that a similar trend was also observed for G178 in the case of purely particle induced turbulence [12]. Comparing the velocity p.d.f. for both G178-R95 and G180-R140 shows also that, as expected, the system with the lowest turbulence intensity will deviate more from the Gaussian distribution than the other one that keeps a nearly Gaussian evolution of the horizontal velocity for fluctuation amplitudes up to five times the standard deviation. We then explore the fluid velocity field in the vicinity of the particles, by looking at the field on the sphere surface $\mathcal{S}^{(i)}$ used for the computation of the local relative velocities in section 3 . Note that the difference in the current analysis is that we look at the very velocity seen by the particles, not at the relative velocity with respect to the particle motion. For more clarity we represented the p.d.f.'s of the velocity fluctuations in linear scale on figure 15, and compared the results with a set of randomly distributed particles. It shows that the particles tend to sample downward-flowing regions, and an estimation of the mean vertical fluid velocity seen by the particle yields $\left\langle u_{z}^{\mathcal{S}_{(i)}}\right\rangle_{p}-\left\langle u_{z}\right\rangle_{\Omega_{f}} \approx-0.072 u_{g}\left(-0.079 u_{g}\right)$ for G178-R95 (G180R140). This point is however difficult to separate from the particles' influence on a meso-scale, since large downward flow events are generated by the wakes of multiple particles in proximity. We have also tested whether particles belonging to a cluster sample the flow differently than the entire set of particles. We observe that particles in clusters seem to avoid regions with the largest velocity amplitude (fig. 9), with a slight shift towards positive $u_{z}$ for G178-M95 (cf. fig. 15). In this case, particles falling through $u_{z}>0$ regions would decelerate, which could be in favour of preferential accumulation but not of an augmentation of the falling speeds. In case G180-R140, the particles in clusters seem to avoid the regions of low velocity amplitude, which can be a sign 
that particles are expelled outside of the vortices. We explored the evolution of $Q$ in the vicinity of the particles (i.e. estimated on the particle-centered spheres $\mathcal{S}_{(i)}$ ) and the corresponding p.d.f.'s show an increase of the probability to sample regions of large strain or large vorticity (fig. 4). This last result indicates that several mechanisms involved are here in competition, with no clear distinction on the dominant one.

\subsection{Influence of two-way coupling}

The precedent considerations tend to propose a scenario to explain how gravity can promote the formation of clusters by shifting the apparent Stokes numbers towards larger values, and filtering by this the influence of the small scale structures in favour of the larger eddies. But this model does not account for any transformation of the eddies induced by the settling. The surrogate Stokes number representative of the largest scales yields for instance $S t_{e}^{(S)}=1.1$ and 0.75 respectively for G178-R95 and G180-R140, which does not necessarily justify the augmentation of $\sigma\left(\mathcal{V}_{\text {Vor }}\right)$ observed from G178-R95 to G180-R140.

However, we have seen that a decrease of the relative turbulence intensity can also affect the coherence of the background turbulence, with a partial destruction of the large scale by the wakes (fig. 6). The aforementioned Stokes numbers refer to the single phase large eddy time scale, but we have seen that the particles tend to increase the dissipation, with the effect of decreasing this large eddy time-scale (assuming that the relation $\tau_{e}^{T P}=u_{r m s}^{T P} / \varepsilon^{T P}$ still holds). As a result, the corresponding time-scales yield $\tau_{e}^{T P} / \tau_{e}^{S P} \approx 0.10$ (0.18) for G178-R95 (G180-R140), which can partially explain the difference observed in the amount of clustering. This destruction can also be the signature of the formation of large-scale columnar flow structures of the pure settling configurations [56], which will further affect the preferential sampling in favor of particle-induced downward-flowing regions. This means that increasing the influence of the settling has two effects in competition: on the one hand it filters the influence of the small scales and enhances the impact of the large scales, on the other hand it disrupts the structure of the large scales, reducing by this their effect on the particle motion. As those effects are difficult to decouple, it would be of interest in the future to systematically vary the turbulence intensity.

\section{Conclusion}

We have simulated the motion of a dilute suspension of fully-resolved, finite-size particles under the combined action of gravity and homogeneous-isotropically forced turbulence in triplyperiodic domains. The solid-to-fluid density ratio was fixed at 1.5 and the Galileo number at approximately 180 which corresponds to a parameter point for which strong wake-induced clustering has been reported in the absence of turbulence [56]. Two simulations have been performed with Taylor-scale Reynolds numbers 95, 140 and particle diameters corresponding to 7, $8.5 \mathrm{Kol}-$ mogorov lengths, respectively. In the absence of gravity, these parameters fall into the range investigated by Uhlmann and Chouippe [55], who have reported slight turbulence-induced particle clustering which has been statistically linked to the properties of the fluid acceleration field. The present work therefore brings the two effects of turbulence and gravity together at parameter points intersecting as much as possible with these earlier studies. The two present simulations differ in their value for the relative turbulence intensity (0.14 and 0.22), which describes the relative importance of these two effects, arguably the principal parameter here.

We find that the average settling velocity (defined as an apparent slip velocity, i.e. averaging separately over each phase and in time, then subtracting both values) is only slightly different from the one for an isolated particle settling in ambient fluid, with an increase by approximately 
$1 \%$ at the lower turbulence intensity and a decrease by $0.6 \%$ for the higher turbulence intensity. At the same time the level of particle clustering, quantified with the aid of Voronoï tessellation analysis, is presently found to be intermediate between the strong clustering of the ambient settling case and the weak clustering of the gravity-free case. This result is not surprising, since forced background turbulence can be expected to interfere with the formation of wake-induced clusters on the different scales of the turbulent spectrum. However, it turns out that the present simulation with the larger value of relative turbulence intensity leads to the larger level of clustering. This means that the tendency to cluster does not monotonously decay as a function of turbulence intensity. As forced background turbulence reduces the level of wake-induced clustering, it simultaneously enables clustering due to preferential concentration. Unfortunately, the limited data which we have available (essentially four data points, cf. figure 11b) does not allow us to further map out the competition between these two opposing effects.

We have analyzed the velocity seen by the particles through sampling and averaging the fluid velocity field on a sphere with radius equal to three particle radii, centered on each particle. It is observed that on average the particles in the present simulations are exposed to more negative vertical fluid velocity than the simple space average. We believe that this result is related to the collective effect of particles having a relatively high probability of being located in the wake region of one or more other particles. On the other hand, the vertical component of the relative velocity computed with the fluid velocity seen by each particle is on average significantly smaller (in amplitude) than the apparent slip velocity, and, therefore, also smaller than the single-particle settling speed (by 5-7\%). A straightforward estimate of the non-linear drag effect (based upon standard quasi-steady drag and the assumption of identically distributed Gaussian fluid velocity fluctuations, as e.g. shown by Homann et al. [27]) predicts a reduction of the settling speed of the order of one percent for the present cases.

Based upon the Voronoï tessellation data we have performed a cluster identification analysis (connecting neighboring Voronoï cells which have a cell volume below a threshold value). We find that the probability distribution of the cluster volumes follows a power-law consistent with the model proposed by Goto and Vassilicos [22] in two space dimensions, as already reported in Uhlmann and Chouippe [55] in the absence of gravity. We note that the same power-law is also exhibited by a random particle distribution, the difference between the various data-sets lying in the tails of the distribution. Most significantly, strong clustering corresponds to much larger probabilities for observing very large clusters. We also observe that forced turbulence has a strong effect upon the geometrical aspect ratio of the clusters: the ratio between the cluster extent in the vertical and the horizontal direction is strongly decreased in both present simulations (as compared to the case without turbulence) for practically all relevant cluster sizes.

Finally we have discussed different mechanisms through which the coherent flow structures are commonly believed to affect the particle motion. The discussion has focused on vortical structures educed via the second invariant of the velocity gradient tensor, and our arguments are based upon visualizations and considerations of the various time scales of the system. We have identified the influence of gravity upon the interaction time scales between a given eddy and a settling particle (at the origin of the crossing trajectory effect) as one potential reason for the different clustering characteristics in our two simulations. The essence is that, as shown by Yoshimoto and Goto [62] for the simplest point-particle model, particles can be expected to interact with a range of flow scales for which a kind of resonance condition is fulfilled, based upon a comparison of time scales (i.e. Stokes numbers). Now taking into account the mean relative velocity (due to settling) leads to a correction of the relevant Stokes number, proportional to the inverse of the relative turbulence intensity. For cases with turbulence intensity below unity, this has the effect of reducing the expected relevance of the small scales for the motion of particles which are larger than the Kolmogorov scale. On the other hand it brings the largest flow scales 
closer towards resonance with the particles by increasing their associated Stokes number. Therefore, under this scenario gravity would further decrease the effect of the smallest flow scales upon particles which are larger than the Kolmogorov scale, and it would promote the effect (beyond pure sweeping) due to the largest scales. This gravity-induced shift of the resonant range of scales might be at the origin of the increase in clustering with turbulence intensity. Other scenarios, however, cannot be excluded as an explanation, such as the influence of the settling particles upon the carrier phase.

As an outlook of the present study we recommend on the one hand to extend the parameter space by performing additional systematic studies varying independently the relative turbulence intensity, the particle diameter and the Galileo number. On the other hand, we believe that it will be highly useful to generate time-resolved series of instantaneous data in order to allow for a detailed analysis of the dynamics of the interaction between particles and coherent structures.

\section{Acknowledgements}

This work was supported by the German Research Foundation (DFG) under project UH 242/12. The simulations were partially performed at LRZ München (under grant pr83la) and at SCC Karlsruhe (project DNSPARTHIT). The computer resources, technical expertise and assistance provided by these centers are thankfully acknowledged.

\section{References}

[1] A. Aliseda, A. Cartellier, F. Hainaux, and J. Lasheras. Effect of preferential concentration on the settling velocity of heavy particles in homogeneous isotropic turbulence. J. Fluid Mech., 468:77-105, 2002. doi: 10.1017/S0022112002001593.

[2] S. Balachandar and J. Eaton. Turbulent dispersed multiphase flow. Ann. Rev. Fluid Mech., 42: 111-133, 2010.

[3] J. Bec, L. Biferale, M. Cencini, A. Lanotte, S. Musacchio, and F. Toschi. Heavy particle concentration in turbulence at dissipative and inertial scales. Phys. Rev. Lett., 98:084502, Feb 2007. doi: 10.1103/PhysRevLett.98.084502.

[4] J. Bec, H. Homann, and S. Ray. Gravity-driven enhancement of heavy particle clustering in turbulent flow. Phys. Rev. Lett., 112:184501, May 2014. doi: 10.1103/PhysRevLett.112.184501.

[5] A. Bragg, P. Ireland, and L. Collins. On the relationship between the non-local clustering mechanism and preferential concentration. J. Fluid Mech., 780:327-343, 2015. doi: 10.1017/ jfm.2015.474.

[6] K. Bürger, M. Treib, R. Westermann, S. Werner, C. C. Lalescu, A. Szalay, C. Meneveau, and G. L. Eyink. Vortices within vortices: hierarchical nature of vortex tubes in turbulence. arXiv preprint arXiv:1210.3325, 2012.

[7] A. Chouippe and M. Uhlmann. Forcing homogeneous turbulence in DNS of particulate flow with interface resolution and gravity. Phys. Fluids, 27(12):123301, 2015. doi: 10.1063/1. 4936274.

[8] J. Chun, D. Koch, S. Rani, A. Ahluwalia, and L. Collins. Clustering of aerosol particles in isotropic turbulence. J. Fluid Mech., 536:219-251, 2005. 
[9] M. Cisse, H. Homann, and J. Bec. Slipping motion of large neutrally buoyant particles in turbulence. Journal of Fluid Mechanics, 735, oct 2013. doi: 10.1017/jfm.2013.490.

[10] S. W. Coleman and J. C. Vassilicos. A unified sweep-stick mechanism to explain particle clustering in two- and three-dimensional homogeneous, isotropic turbulence. Physics of Fluids, 21(11):113301, nov 2009. doi: 10.1063/1.3257638.

[11] G. T. Csanady. Turbulent diffusion of heavy particles in the atmosphere. Journal of the Atmospheric Sciences, 20(3):201-208, may 1963. doi: 10.1175/1520-0469(1963)020<0201:tdohpi $>2$. $0 . c 0 ; 2$.

[12] T. Doychev. The dynamics of finite-size settling particles. PhD thesis, Karlsruhe Institute of Technology, 2014.

[13] V. Eswaran and S. Pope. An examination of forcing in direct numerical simulations of turbulence. 16:257-278, 1988. ISSN 00457930. doi: 10.1016/0045-7930(88)90013-8.

[14] J.-S. Ferenc and Z. Neda. On the size distribution of Poisson Voronoi cells. Physica A, 385: 518-526, 2007.

[15] A. Ferrante and S. Elghobashi. On the physical mechanisms of two-way coupling in particleladen isotropic turbulence. Phy. Fluids, 15(2):315-329, 2003.

[16] L. Fiabane, R. Zimmermann, R. Volk, J.-F. Pinton, and M. Bourgoin. Clustering of finite-size particles in turbulence. Phys. Rev. E, 86(035301(R)), 2012.

[17] W. Fornari, F. Picano, and L. Brandt. Sedimentation of finite-size spheres in quiescent and turbulent environments. J. Fluid Mech., 788:640-669, 2016. doi: 10.1017/jfm.2015.698.

[18] W. Fornari, F. Picano, G. Sardina, and L. Brandt. Reduced particle settling speed in turbulence. J. Fluid Mech., 808:153-167, 2016. doi: 10.1017/jfm.2016.648.

[19] A. Fortes, D. Joseph, and T. Lundgren. Nonlinear mechanics of fluidization of beds of spherical particles. J. Fluid Mech., 177:467-483, 1987.

[20] R. Glowinski, T.-W. Pan, T. Hesla, and D. Joseph. A distributed Lagrange multiplier/fictitious domain method for particulate flows. Int. J. Multiphase Flow, 25:755-794, 1999.

[21] G. Good, P. Ireland, G. Bewley, E. Bodenschatz, L. Collins, and Z. Warhaft. Settling regimes of inertial particles in isotropic turbulence. J. Fluid Mech., 759:R3, 2014. doi: 10.1017/jfm. 2014.602.

[22] S. Goto and J. Vassilicos. Self-similar clustering of inertial particles and zero-acceleration points in fully developed two-dimensional turbulence. Phys. Fluids, 18(11):115103, 2006.

[23] S. Goto and J. Vassilicos. Sweep-stick mechanism of heavy particle clustering in fluid turbulence. Phys. Rev. Lett., 100(5):054503, 2008.

[24] W. Grabowski and L.-P. Wang. Growth of cloud droplets in a turbulent environment. Annu. Rev. Fluid Mech., 45(1):293-324, 2013. doi: 10.1146/annurev-fluid-011212-140750.

[25] K. Gustavsson and B. Mehlig. Statistical models for spatial patterns of heavy particles in turbulence. Adv. Phys., 65(1):1-57, 2016. doi: 10.1080/00018732.2016.1164490. 
[26] R. Hogan and J. Cuzzi. Stokes and Reynolds number dependence of preferential particle concentration in simulated three-dimensional turbulence. Phys. Fluids, 13(10):2938-2945, 2001. doi: http://dx.doi.org/10.1063/1.1399292.

[27] H. Homann, J. Bec, and R. Grauer. Effect of turbulent fluctuations on the drag and lift forces on a towed sphere and its boundary layer. J. Fluid Mech., 721:155-179, 4 2013. ISSN 14697645. doi: $10.1017 / \mathrm{jfm} .2013 .66$.

[28] S. G. Huisman, T. Barois, M. Bourgoin, A. Chouippe, T. Doychev, P. Huck, C. E. Bello Morales, M. Uhlmann, and R. Volk. Columnar structure formation of a dilute suspension of settling spherical particles in a quiescent fluid. Phys. Rev. Fluids, 1:074204, Nov 2016. doi: 10.1103/PhysRevFluids.1.074204.

[29] J. Hunt, A. Wray, and P. Moin. Eddies, streams, and convergence zones in turbulent flows. In Proceedings of the Summer Programm, pages 193-208. (Center for Turbulence Research, Stanford), 1988.

[30] W. Hwang and J. K. Eaton. Homogeneous and isotropic turbulence modulation by small heavy $(S t \sim 50)$ particles. J. Fluid Mech., 564:361, 2006. ISSN 0022-1120. doi: 10.1017/ S0022112006001431.

[31] P. Ireland, A. Bragg, and L. Collins. The effect of Reynolds number on inertial particle dynamics in isotropic turbulence. Part 2. Simulations with gravitational effects. J. Fluid Mech., 796:659-711, 2016. doi: 10.1017/jfm.2016.227.

[32] M. Jenny, J. Dušek, and G. Bouchet. Instabilities and transition of a sphere falling or ascending freely in a Newtonian fluid. J. Fluid Mech., 508:201-239, 2004. ISSN 0022-1120. doi: 10.1017/S0022112004009164.

[33] T. Kajishima. Influence of particle rotation on the interaction between particle clusters and particle-induced turbulence. Int. J. Heat Fluid Flow, 25(5):721-728, 2004.

[34] T. Kajishima and S. Takiguchi. Interaction between particle clusters and particle-induced turbulence. Int. J. Heat Fluid Flow, 23:639-646, 2002.

[35] A. Kidanemariam, C. Chan-Braun, T. Doychev, and M. Uhlmann. Direct numerical simulation of horizontal open channel flow with finite-size, heavy particles at low solid volume fraction. New J. Phys., 15, 2013. ISSN 13672630. doi: 10.1088/1367-2630/15/2/025031.

[36] M. Lance and J. Bataille. Turbulence in the liquid phase of a uniform bubbly air-water flow. J. Fluid Mech., 222:95-118, 1991.

[37] M. Maxey. The gravitational settling of aerosol particles in homogeneous turbulence and random flow fields. J. Fluid Mech., 174:441-465, 1987.

[38] R. Monchaux and A. Dejoan. Settling velocity and preferential concentration of heavy particles under two-way coupling effects in homogeneous turbulence. Phys. Rev. Fluids, 2:104302, Oct 2017. doi: 10.1103/PhysRevFluids.2.104302.

[39] R. Monchaux, M. Bourgoin, and a. Cartellier. Preferential concentration of heavy particles: A Voronoï analysis. Phys. Fluids, 22(10):103304, 2010. ISSN 10706631. doi: 10.1063/1.3489987.

[40] R. Monchaux, M. Bourgoin, and A. Cartellier. Analyzing preferential concentration and clustering of inertial particles in turbulence. Int. J. Multiphase Flow, 40:1-18, 2012. 
[41] P. Nielsen. Turbulence effects on the settling of suspended particles. J. Sed. Res., 63(5):835838, 1993. doi: 10.1306/D4267C1C-2B26-11D7-8648000102C1865D.

[42] M. Obligado, T. Teitelbaum, A. Cartellier, P. Mininni, and M. Bourgoin. Preferential concentration of heavy particles in turbulence. Journal of Turbulence, 15(5):293-310, 2014. doi: 10.1080/14685248.2014.897710.

[43] B. Oesterlé. A note on crossing-trajectory effects in gas-particle turbulent flows. In Computational Methods in Multiphase Flow IV. WIT Press, may 2007. doi: 10.2495/mpf070371.

[44] G. Riboux, F. Risso, and D. Legendre. Experimental characterization of the agitation generated by bubbles rising at high reynolds number. Journal of Fluid Mechanics, 643:509-539, 2010. doi: 10.1017/S0022112009992084.

[45] F. Risso. Theoretical model for $k^{-3}$ spectra in dispersed multiphase flows. Phys. Fluids, 23 (1):011701, 2011. doi: 10.1063/1.3530438.

[46] B. Rosa, H. Parishani, O. Ayala, and L.-P. Wang. Settling velocity of small inertial particles in homogeneous isotropic turbulence from high-resolution DNS. Int. J. Multiphase Flow, 83: 217-231, 2016. doi: 10.1016/j.ijmultiphaseflow.2016.04.005.

[47] L. Schiller and A. Naumann. A drag coefficient correlation. VDI Zeitung, 77(318):51, 1935.

[48] R. Shaw. Particle-turbulence interactions in atmospheric clouds. Annu. Rev. Fluid Mech., 35 (1):183-227, 2003. doi: 10.1146/annurev.fluid.35.101101.161125.

[49] K. Squires and J. Eaton. Preferential concentration of particles by turbulence. Phys. Fluids A, 3(5):1169-1178, 1991.

[50] S. Sumbekova, A. Cartellier, A. Aliseda, and M. Bourgoin. Preferential concentration of inertial sub-Kolmogorov particles. The roles of mass loading of particles, Stokes and Reynolds numbers. Phys. Rev. Fluids, 2(2):024302, 2016. doi: 10.1103/PhysRevFluids.2.024302.

[51] T. Tanaka and J. K. Eaton. Classification of turbulence modification by dispersed spheres using a novel dimensionless number. Phys. Rev. Lett., 101(September):1-4, 2008. ISSN 00319007. doi: 10.1103/PhysRevLett.101.114502.

[52] E. Tunstall and G. Houghton. Retardation of falling spheres by hydrodynamic oscillations. Chem. Eng. Sci., 23(9):1067-1081, 1968.

[53] M. Uhlmann. An immersed boundary method with direct forcing for the simulation of particulate flows. Journal of Computational Physics, 209:448-476, 2005. ISSN 00219991. doi: 10.1016/j.jcp.2005.03.017.

[54] M. Uhlmann. Interface-resolved direct numerical simulation of vertical particulate channel flow in the turbulent regime. Phys. Fluids, 20(May 2013), 2008. doi: 10.1063/1.2912459.

[55] M. Uhlmann and A. Chouippe. Clustering and preferential concentration of finite-size particles in forced homogeneous-isotropic turbulence. J. Fluid Mech., 812:991-1023, 2017. doi: $10.1017 /$ jfm.2016.826.

[56] M. Uhlmann and T. Doychev. Sedimentation of a dilute suspension of rigid spheres at intermediate Galileo numbers: the effect of clustering upon the particle motion. J. Fluid Mech., 752:310-348, 2014. doi: 10.1017/jfm.2014.330. 
[57] M. Uhlmann and J. Dušek. The motion of a single heavy sphere in ambient fluid: A benchmark for interface-resolved particulate flow simulations with significant relative velocities. International Journal of Multiphase Flow, 59:221-243, 2014. ISSN 03019322. doi: 10.1016/j.ijmultiphaseflow.2013.10.010.

[58] L.-P. Wang and M. Maxey. Settling velocity and concentration distribution of heavy particles in homogeneous isotropic turbulence. J. Fluid Mech., 256:27-68, 1993.

[59] M. R. Wells and D. E. Stock. The effects of crossing trajectories on the dispersion of particles in a turbulent flow. Journal of Fluid Mechanics, 136:31-62, 1983. doi: 10.1017/ S0022112083002049.

[60] J. Wu and R. Manasseh. Dynamics of dual-particles settling under gravity. Int. J. Multiphase Flow, 24:1343-1358, 1998.

[61] T. Yang and S. Shy. The settling velocity of heavy particles in an aqueous near-isotropic turbulence. Phys. Fluids, 15(4):868-880, 2003. doi: 10.1063/1.1557526.

[62] H. Yoshimoto and S. Goto. Self-similar clustering of inertial particles in homogeneous turbulence. J. Fluid Mech., 577:275-286, 2007.

[63] M. Yudine. Physical considerations on heavy-particle diffusion. volume 6 of Advances in Geophysics, pages 185 - 191. Elsevier, 1959.

[64] L. Zaichik and V. Alipchenkov. Pair dispersion and preferential concentration of particles in isotropic turbulence. Phys. Fluids, 15(6):1776-1787, 2003.

[65] L. Zaichik and V. Alipchenkov. Refinement of the probability density function model for preferential concentration of aerosol particles in isotropic turbulence. Phys. Fluids, 19(11): 113308, 2007. 
(b)

(a)

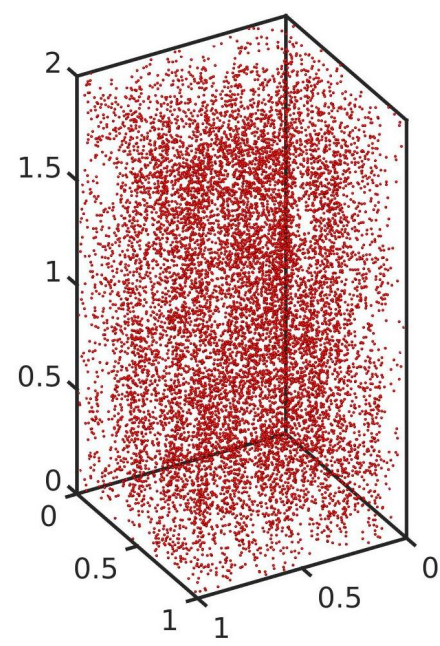

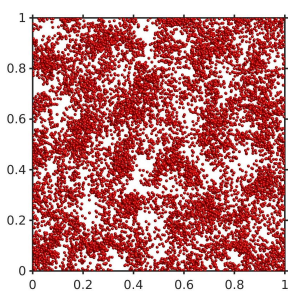

(c)

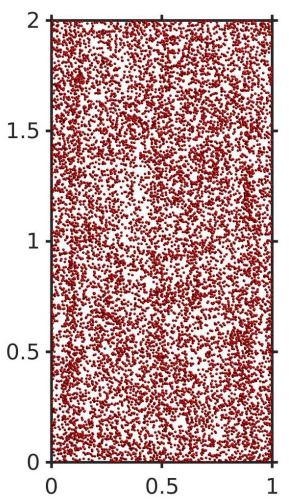

(d)

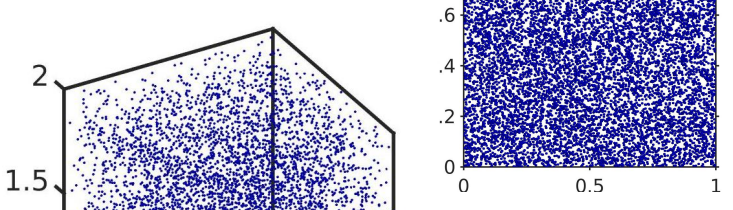

(f)

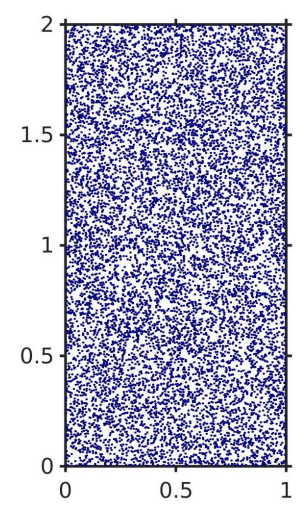

Figure 1: Visualization of particle positions for both types of initial state used in the current study: (a-c) columns (corresponding to the final state of the case G178) used in G178-R95, (d-f) Randomly distributed particles used in G180-R140.

(a)

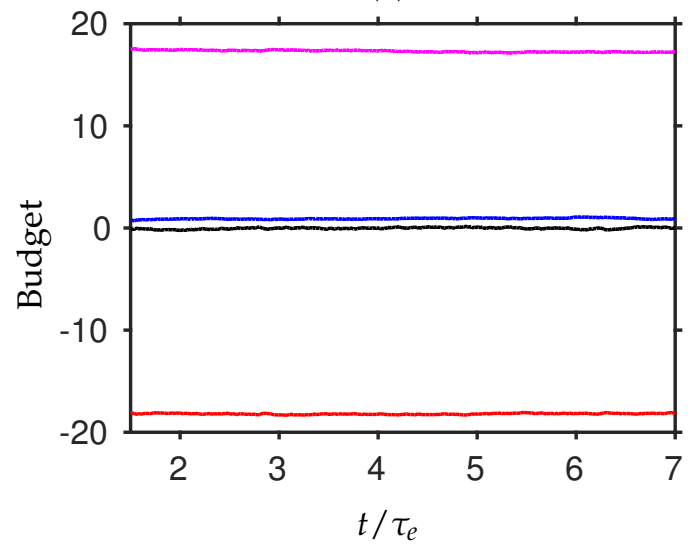

(b)

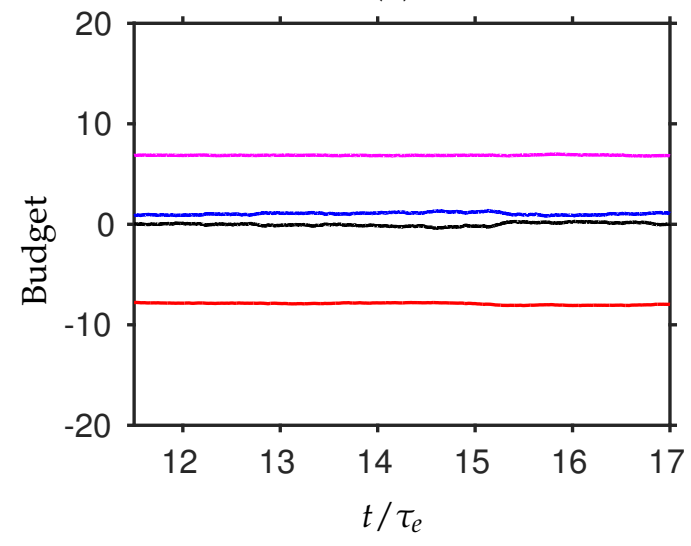

Figure 2: Time evolution of the different terms in the budget of the volume averaged kinetic energy: $0=$ $-\mathrm{d}\left\langle E_{k}\right\rangle_{\Omega} / \mathrm{d} t-\varepsilon_{\Omega}+\Psi^{(t)}+\Psi^{(p)}$ scaled by the dissipation of the corresponding single phase simulation for case G178-R95 (a) and G180-R140 (b). Linestyle: $-\left(-\mathrm{d}\left\langle E_{k}\right\rangle_{\Omega} / \mathrm{d} t\right),-\left(-\varepsilon_{\Omega}\right),-\Psi^{(t)},-\Psi^{(p)}$ 
(a)

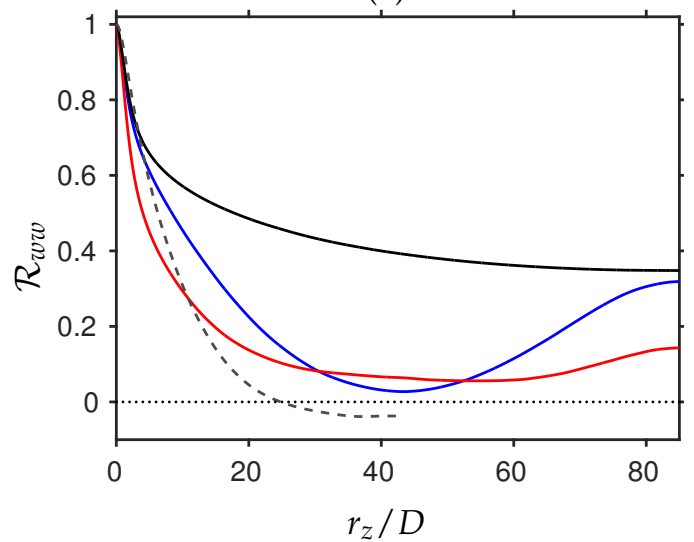

Figure 3: (a) Longitudinal two-point correlation function in the z-coordinate direction, $\mathcal{R}_{w w}\left(r_{z}\right)$. Linestyle: - G178-R95, - G180-R140, — G178, --- R95

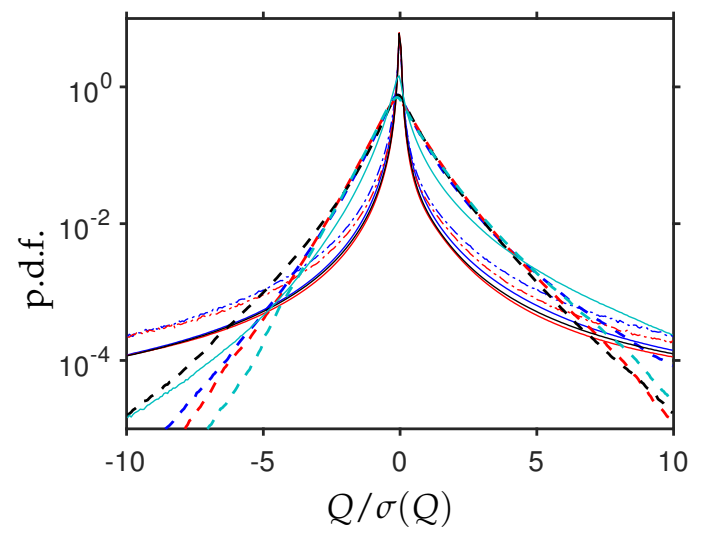

\begin{tabular}{rcc}
\hline case & $\sigma(Q) / \omega_{r m s}$ & $\sigma\left(Q^{\text {filt }}\right) / \omega_{r m s}$ \\
\hline G178-R95 & $2.96 \times 10^{1}$ & $6.59 \times 10^{-1}$ \\
G180-R140 & $6.22 \times 10^{1}$ & 2.66 \\
G178 & $3.07 \times 10^{1}$ & $6.23 \times 10^{-1}$ \\
R95 & $1.49 \times 10^{1}$ & $1.95 \times 10^{-1}$ \\
\hline
\end{tabular}

Figure 4: P.d.f. of the second invariant of the velocity gradient tensor, $Q$, [29] for the unfiltered field (solid lines) and the filtered field (dashed lines). The field has been filtered with a box-filter of width $\Delta_{\text {filt }}=$ $89 \Delta x$ for the particle-laden cases (corresponding to $\Delta_{\text {filt }}=3.7 \mathrm{D}$ for the settling cases and $\Delta_{\text {filt }}=5.6 \mathrm{D}$ for G0-R120) and $\Delta_{\text {filt }}=45 \Delta x$ for the single phase case such as to keep the same ratio $\Delta_{\text {filt }} / \eta^{S P}$ as G178R95. The dashed-dotted lines correspond to the values of $Q$ sampled on the spheres $\mathcal{S}_{(i)}$ centered on the particles. Linestyle: — G180-R140, — G178-R95, — G178, - R95. The table lists the standard deviation of $Q$ computed for the unfiltered field $(Q)$ and the filtered field $\left(Q^{\text {filt }}\right)$. 
(a)

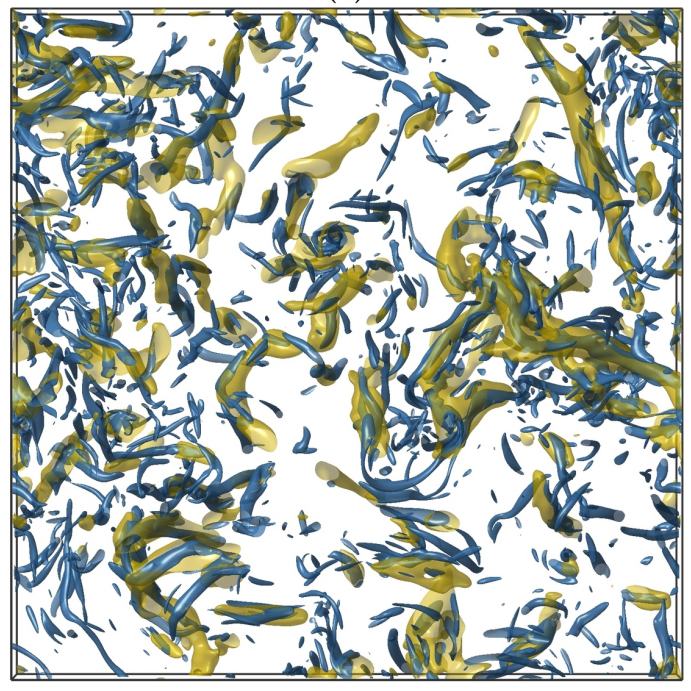

(b)

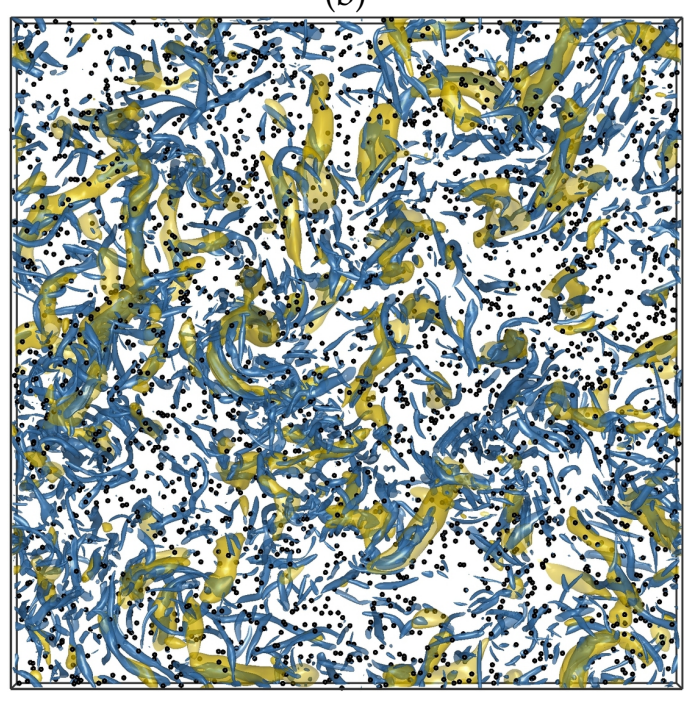

(c)

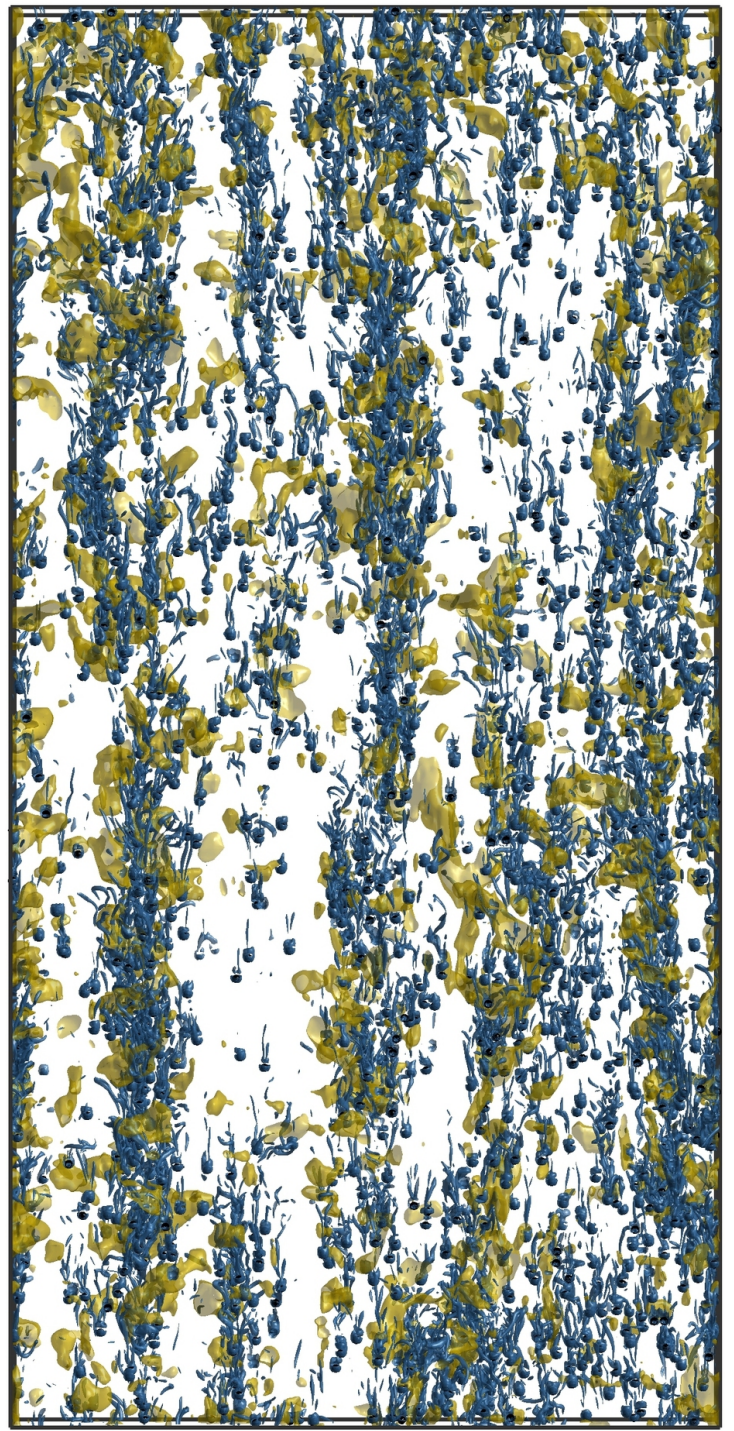

Figure 5: Isocontour of $Q$ for the unfiltered field (blue) and the filtered field (yellow) for the single phase case R95 (a), the case G0-R120 (b) and the case G178 (d). The filter width is equal to $\Delta_{f i l t}=89 \Delta x$ for both cases G0-R114 and G178, and to $\Delta_{\text {filt }}=45 \Delta x$ for R95. For the unfiltered field the isocontour corresponds to $Q=1.5 \sigma(Q)$ in the absence of gravity and $Q=0.5 \sigma(Q)$ for M178, while the filtered field corresponds to $Q_{\text {filt }}=1.5 \sigma\left(Q_{\text {filt }}\right)$. For the three figures the visualizations represent one eighth of the domain in the depth (into the page) and the total domain in the other two directions. 
(a)

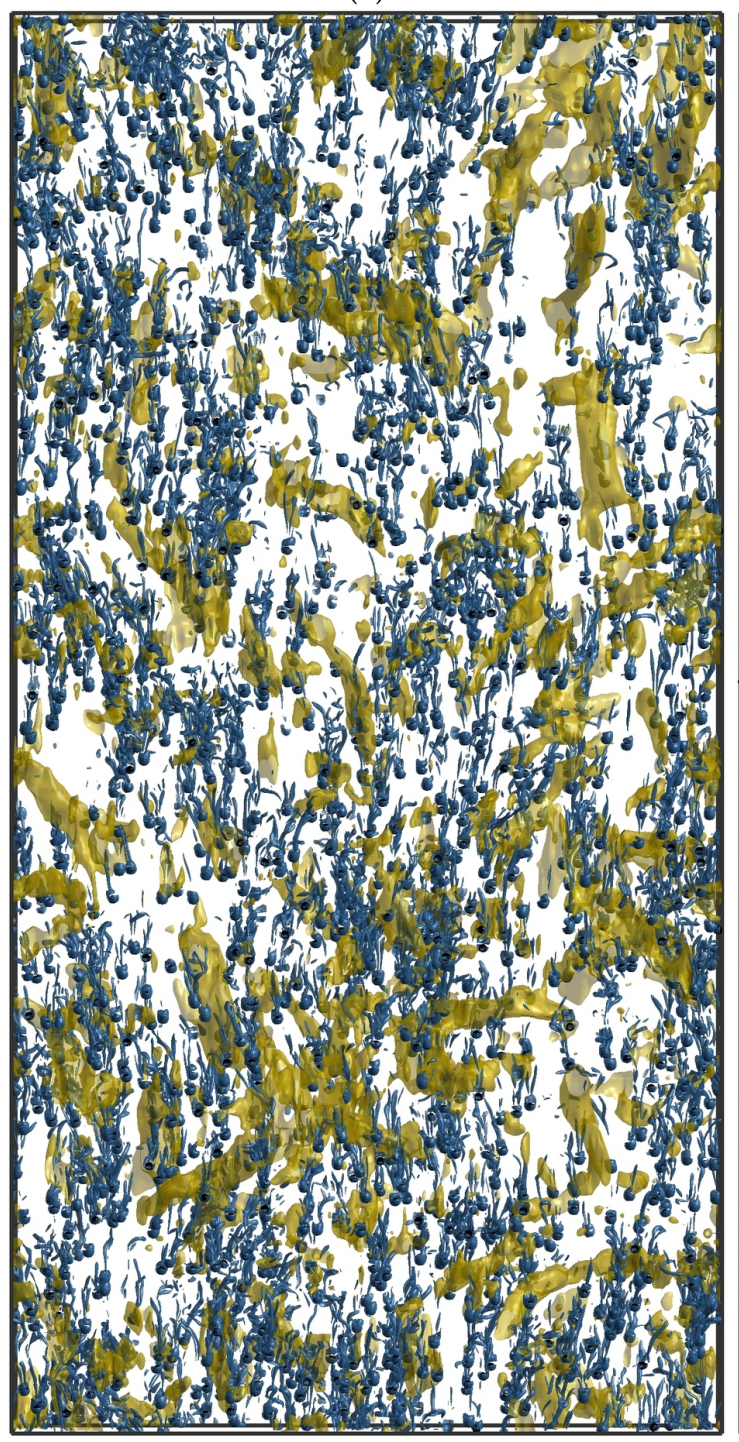

(b)

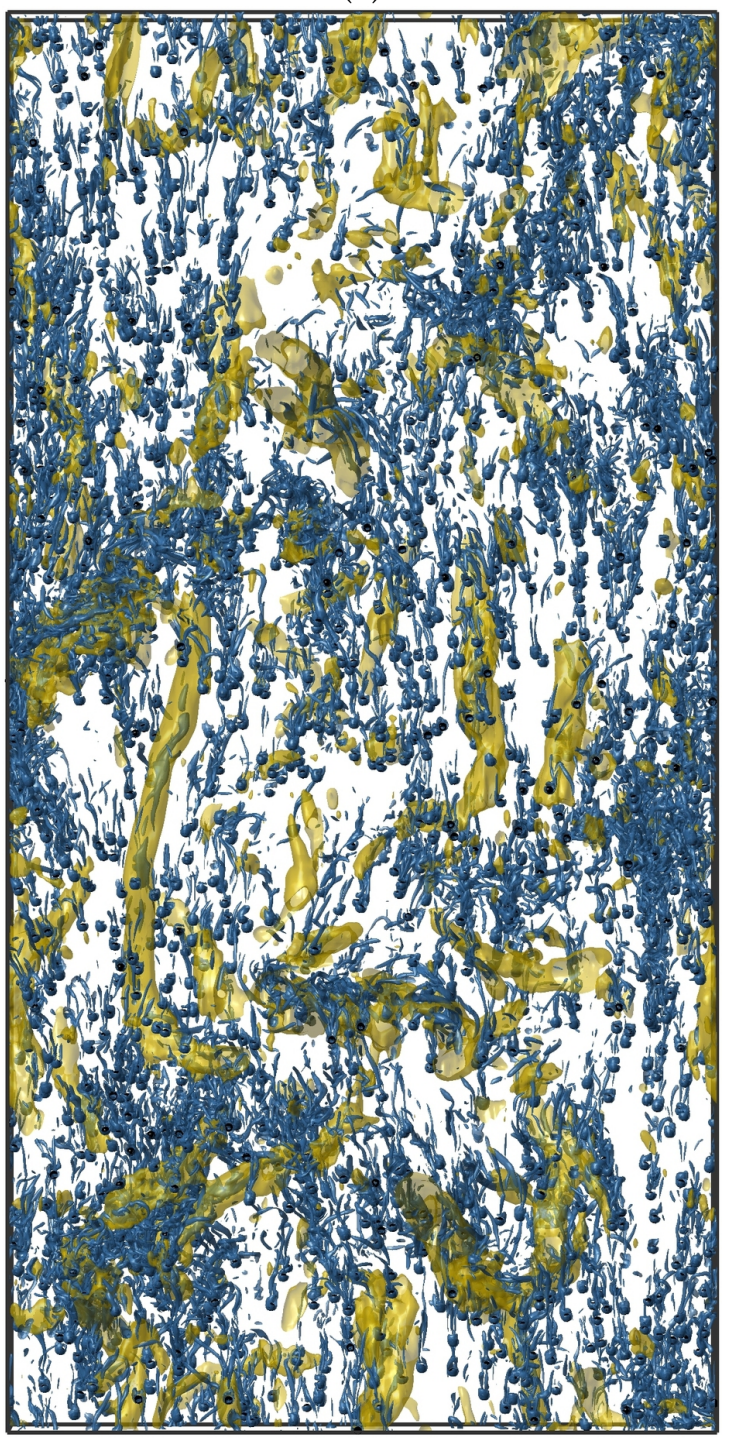

Figure 6: Isocontour of the $\mathrm{Q}$ criterion for the unfiltered field (blue) and the filtered field (yellow) for the case M178-R95 (a) and G180-R140 (b). The filter width is equal to $\Delta_{f i l t}=89 \Delta x$ for both cases. For the unfiltered field the isocontour corresponds to $Q=0.5 \sigma(Q)$ while the filtered field corresponds to $Q_{\text {filt }}=1.5 \sigma\left(Q_{\text {filt }}\right)$. For both figures the visualizations represent one eighth of the domain in the depth (into the page) direction and the total domain in the other two directions. 
(a)

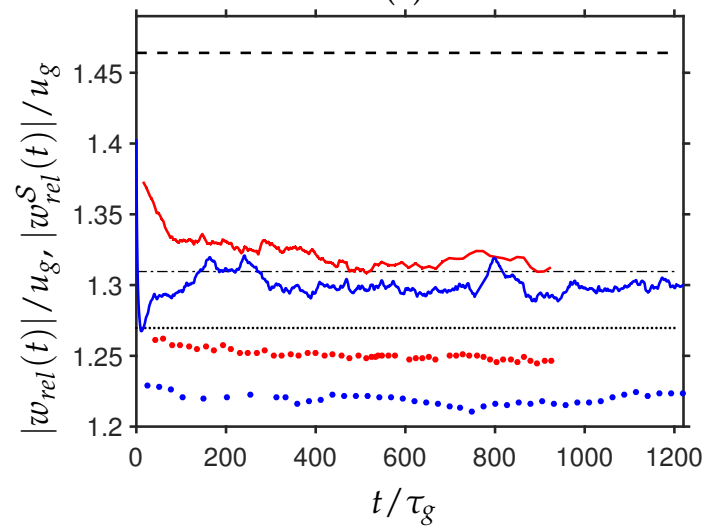

(b)

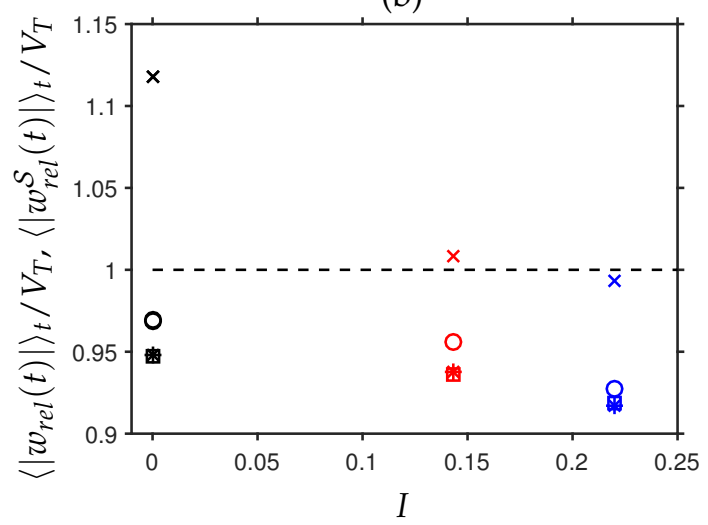

Figure 7: (a) Time evolution of the settling velocity (continuous and dashed lines: $w_{r e l}=\left\langle u_{r e l, z}^{(i)}\right\rangle_{p}$, dots: $w_{r e l}^{\mathcal{S}}=\left\langle u_{r e l, z}^{\mathcal{S}_{(i)}}\right\rangle_{p}$ (cf. equ. 16), - - one single particle in ambient flow, --- mean settling velocity for case G178, $\cdots$ mean settling velocity based on the velocity seen by the particles $\left(w_{\text {rel }}^{\mathcal{S}}\right)$ for case G178 (note that for G178 the two definitions correspond to the average both in time and over the set of particles). (b) Time average of the mean settling velocity as a function of the turbulence intensity $I=u_{r m s}^{S P} / V_{T}$. The crosses correspond to the apparent relative velocity $w_{r e l}$ and circles to the relative velocity based upon the sphere averaging procedure $w_{r e l}^{\mathcal{S}}$, square to $w_{r e l, f 1}^{\mathcal{S}}$ and stars to $w_{r e l, f 2}^{\mathcal{S}}$. Linestyle: - G178-R95, - G180-R140, - G178

(a)

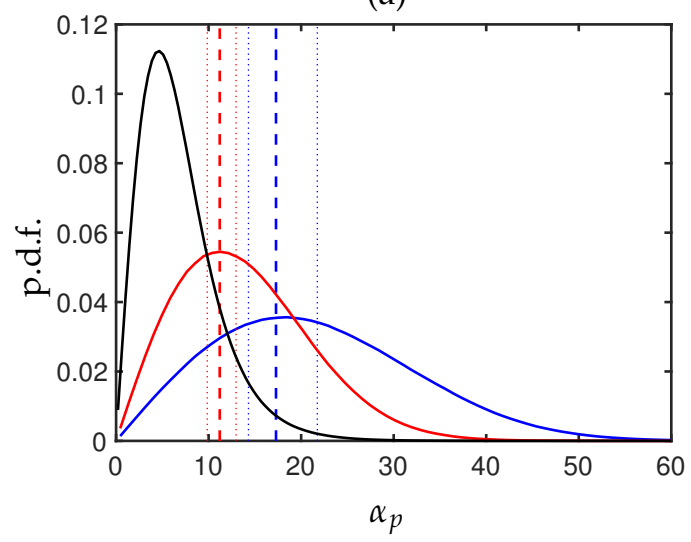

(b)

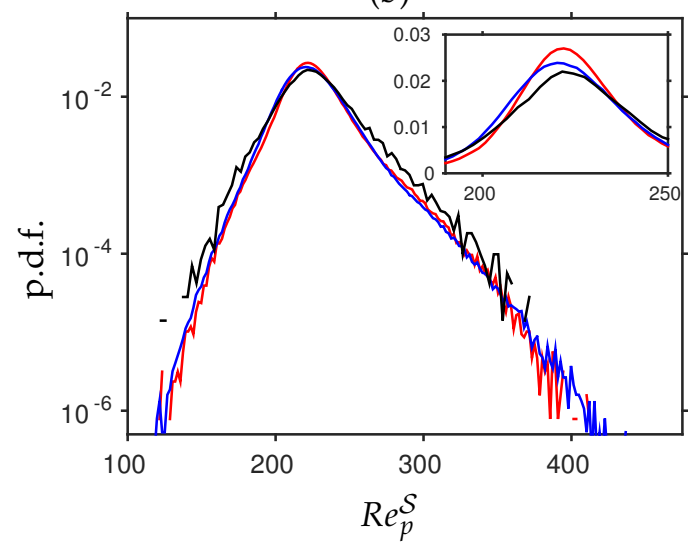

Figure 8: (a) P.d.f. of the angle $\alpha_{p}$ between the relative particle velocity vector and the vertical axis (in degrees). The vertical lines indicate the location of the reference angles $\alpha_{r e f}^{i}$ defined as $\tan \left(\alpha_{r e f}^{i}\right)=$ $\left(2 u_{r m s}^{S P}\right)^{1 / 2} /\left|V_{T}+i \times u_{r m s}^{S P}\right|$. The dashed lines corresponds to $\alpha_{r e f}^{0}$ and the dotted lines to $\alpha_{r e f}^{ \pm 1}$ (we have $\alpha_{r e f}^{-1}<\alpha_{r e f}^{0}<\alpha_{r e f}^{1}$ ). (b) P.d.f. of the Reynolds number of the particles based on the shell averaged relative velocities: $\operatorname{Re}_{p}^{\mathcal{S}_{(i)}}=D\left|\mathbf{u}_{r e l}^{\mathcal{S}_{(i)}}\right| / v$. Linestyle: - G180-R140, — G178-R95, - G178. The inset shows the same data in linear scale. 
(a)

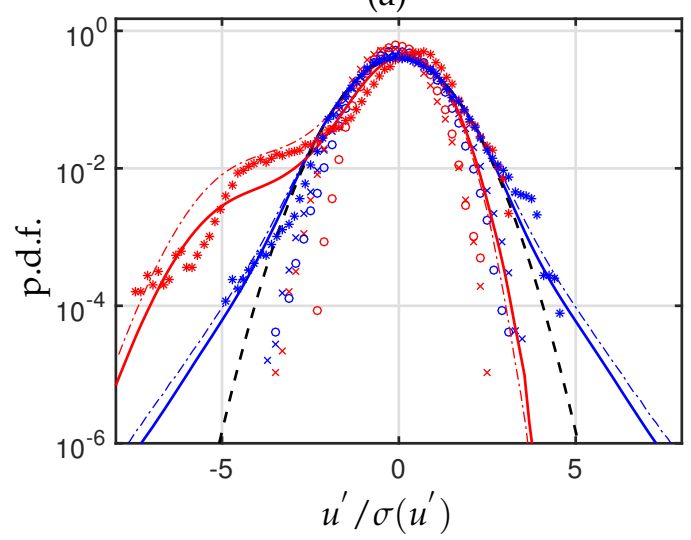

(b)

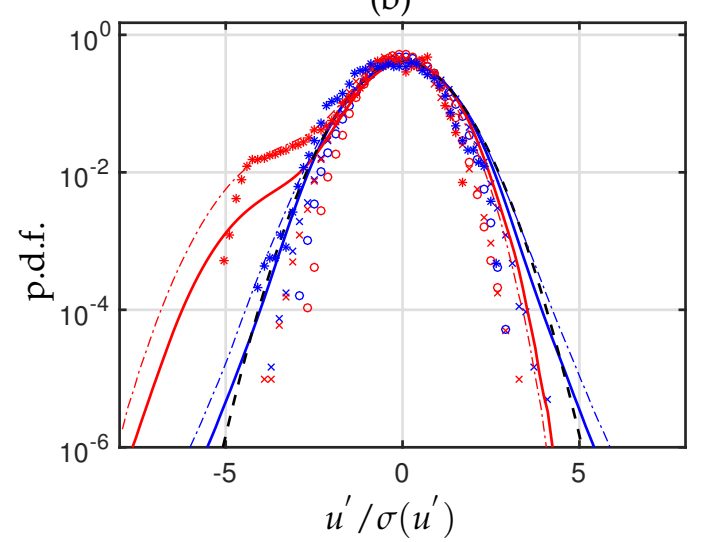

Figure 9: P.d.f. of the fluid velocity computed on different regions of the flow for the case (a) G178-R95 and (b) G180-R140. Continuous lines represent the full domain occupied by the fluid $\Omega_{f}$, dashed-dotted lines refer to the flow regions sampled by the spheres $\mathcal{S}_{(i)}$ used for the computation of the local relative velocity. The stars correspond to sampling on the particle-centered spheres of those particles which are members of a cluster. Crosses refer to the velocities obtained when sampling over the particle-centered spheres, and then averaging; the open circles are data from samples on the surface of randomly-placed spheres, and then averaging. The dashed line indicates the Gaussian distribution. Colorstyle: — vertical component, horizontal component.

(a)

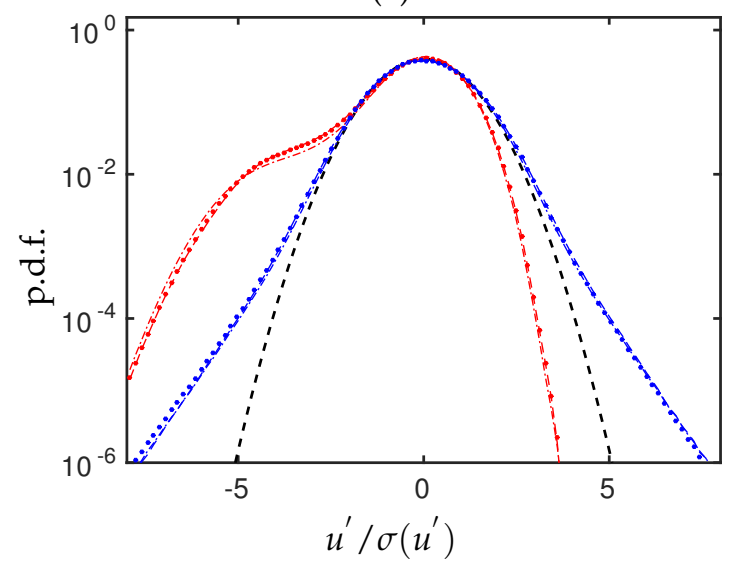

(b)

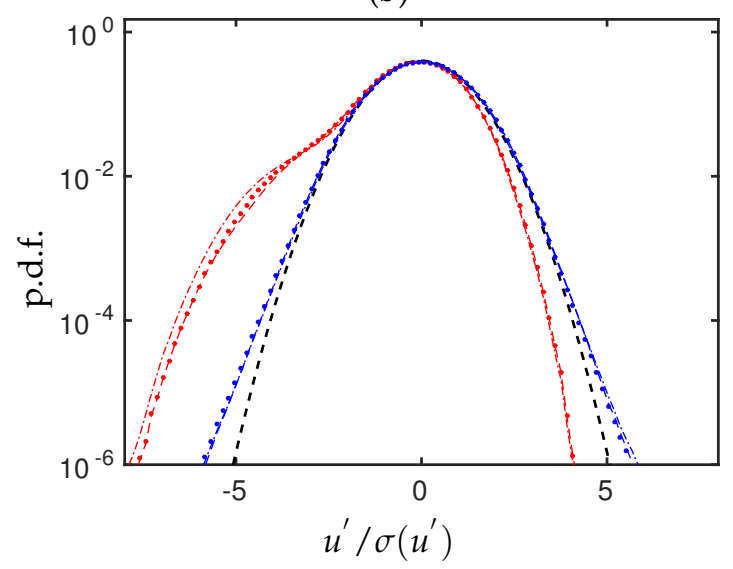

Figure 10: P.d.f. of the fluid velocity computed on different regions of the flow for the case (a) G178-R95 and (b) G180-R140. Dashed-dotted lines refer to the flow regions sampled by the spheres $\mathcal{S}_{(i)}$ used for the computation of the local relative velocity, dashed lines to the velocities sampled at the front of the spheres according to the first definition and the dotted lines to the front of the spheres according to the second definition. The black dashed line remind the Gaussian distribution. Colorstyle: — vertical component, horizontal component. 
(a)

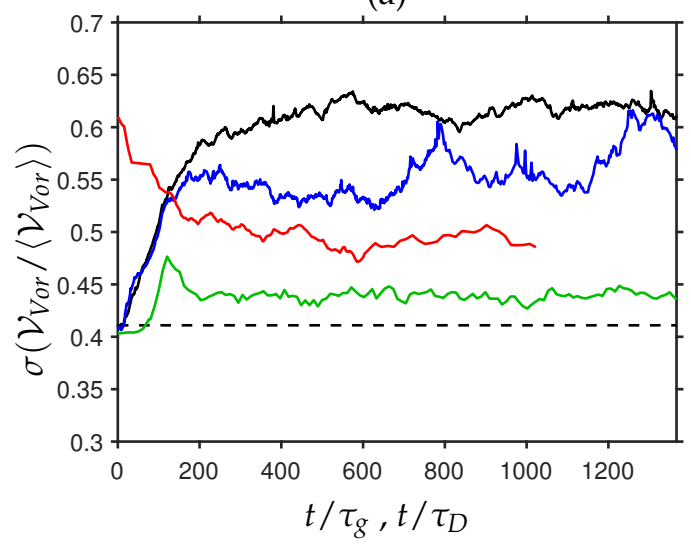

(b)

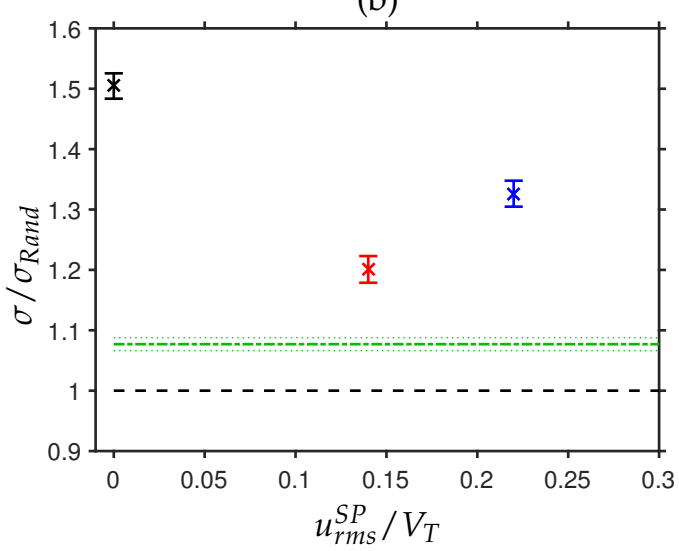

Figure 11: (a) Time evolution of the standard deviation of the volume of the Voronoï cells. For the cases G180-R140, G178-R95 and G178 time is scaled by $\tau_{g}$, for the case G0-R120 as gravity is set to zero we used the time scale $\tilde{\tau}_{D}=D / u_{r m s}^{S P}$ for the scaling. (b) Time-mean of the standard deviation in (a) scaled by its equivalent for a corresponding set of randomly distributed particles, plotted as a function of the relative turbulence intensity. The crosses indicates the cases with settling, the green dash-dotted line the case G0R114, and the dashed line the random reference. Linestyle: — G178-R95, — G180-R140, — G178, — G0R120, --- randomly distributed particles.

(a)

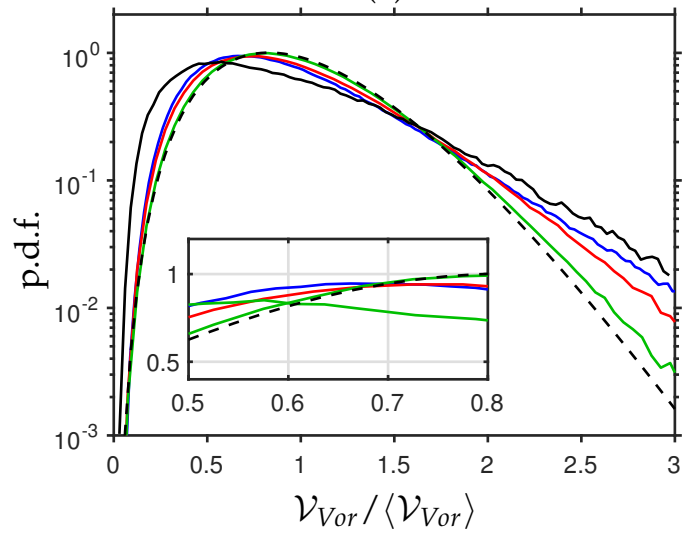

Figure 12: (a) P.d.f. of the volume of the Voronoï cells. The inset shows a close-up of the same data around the lower cross-over points. Linestyle: — G178-R95, — G180-R140, — G178, — G0-R120, --- randomly distributed finite-size particles. 
(a)

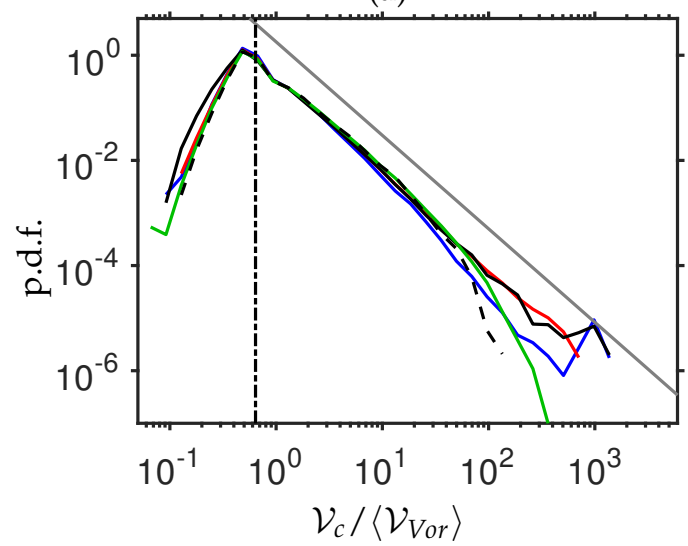

(b)

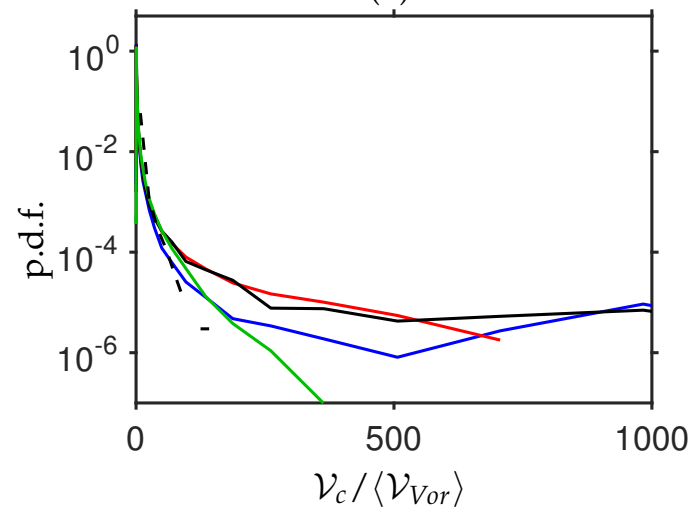

Figure 13: P.d.f. of the cluster volume $\mathcal{V}_{c}$ in logarithmic scale (a) and in linear scale (b). Linestyle: - G178R95, — G180-R140, — G178, — G0-R120, --- randomly distributed particles. In (a) the vertical dash-dotted line indicates the clustering threshold volume, $\mathcal{V}_{\text {Vor }}^{\text {clus }}$, the dark grey line is proportional to $\mathcal{V}_{c}^{-16 / 9}$.

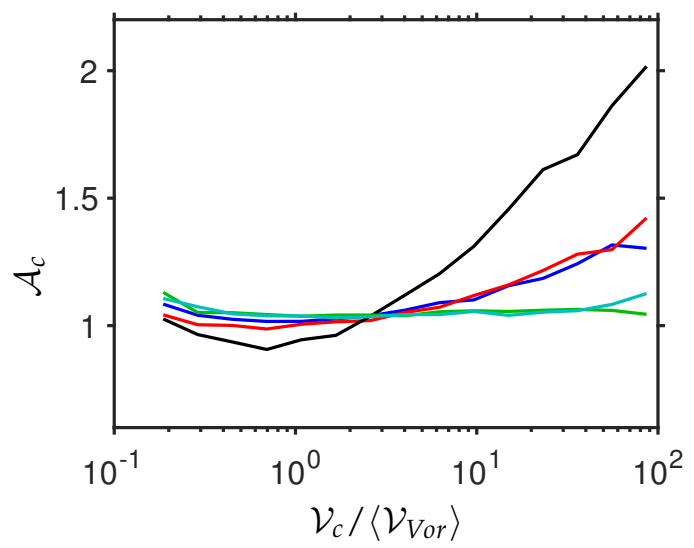

Figure 14: Mean geometrical aspect ratio $\mathcal{A}_{c}=L_{c}^{z} / L_{c}^{x, y}$ of the clusters plotted as a function of the cluster volume. — G178-R95, — G180-R140, — G178, — G0-R120, — randomly distributed particles. 
(a)

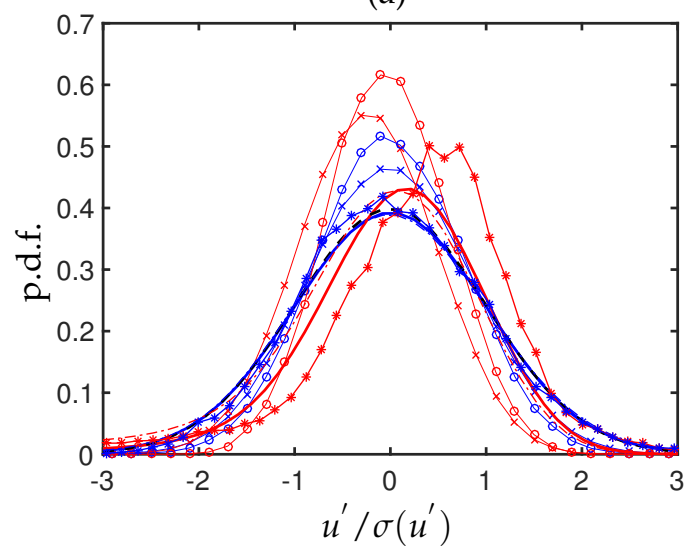

(b)

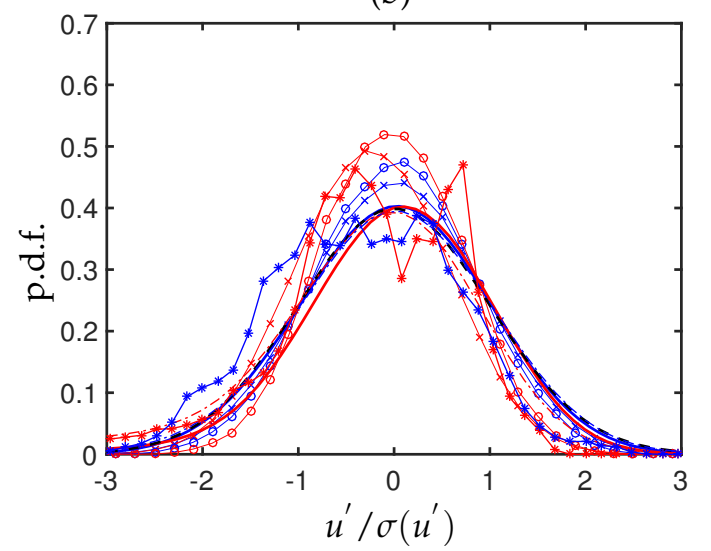

Figure 15: P.d.f. of the fluid velocity (as in fig. 9), but here plotted in linear scale for case (a) G178-R95 and (b) G180-R140. Continuous lines represent the full domain occupied by the fluid $\Omega_{f}$, dash-dotted lines refer to the flow regions sampled by the spheres $\mathcal{S}_{(i)}$ used for the computation of the local relative velocity. The stars correspond to sampling on the particle-centered spheres of those particles which are members of a cluster. Crosses refer to the velocities obtained when sampling over the particle-centered spheres, and then averaging; the open circles are data from samples on the surface of randomly-placed spheres, and then averaging. The dashed line indicates the Gaussian distribution. Colorstyle: — vertical component, horizontal component. 